\title{
On the continuity of mean total normal stress in geometrical multiscale cardiovascular problems
}

\author{
Pablo J. Blanco ${ }^{\mathrm{a}, \mathrm{b}}$, Simone Deparis ${ }^{\mathrm{c}}$, A. Cristiano I. Malossi ${ }^{\mathrm{c}, *}$ \\ a LNCC, Laboratório Nacional de Computação Científica, Av. Getúlio Vargas 333, Quitandinha, 25651-075 Petrópolis, Brazil \\ ${ }^{\mathrm{b}}$ INCT-MACC, Instituto Nacional de Ciência e Tecnologia em Medicina Assistida por Computação Científica, Petrópolis, Brazil \\ ${ }^{\mathrm{c}}$ CMCS, Chair of Modeling and Scientific Computing, MATHICSE, Mathematics Institute of Computational Science and Engineering, EPFL, École Polytechnique \\ Fédérale de Lausanne, Station 8, CH-1015 Lausanne, Switzerland
}

\section{A R T I C L E I N F O}

\section{Article history:}

Received 22 December 2012

Received in revised form 18 April 2013

Accepted 26 May 2013

Available online 10 June 2013

\section{Keywords:}

Dimensionally-heterogeneous models

Coupling conditions

Geometrical multiscale modeling

Total stress boundary data

Fluid-structure interaction

\begin{abstract}
A B S T R A C T
In this work an iterative strategy to implicitly couple dimensionally-heterogeneous blood flow models accounting for the continuity of mean total normal stress at interface boundaries is developed. Conservation of mean total normal stress in the coupling of heterogeneous models is mandatory to satisfy energetic consistency between them. Nevertheless, existing methodologies are based on modifications of the Navier-Stokes variational formulation, which are undesired when dealing with fluid-structure interaction or black box codes. The proposed methodology makes possible to couple one-dimensional and threedimensional fluid-structure interaction models, enforcing the continuity of mean total normal stress while just imposing flow rate data or even the classical Neumann boundary data to the models. This is accomplished by modifying an existing iterative algorithm, which is also able to account for the continuity of the vessel area, when required. Comparisons are performed to assess differences in the convergence properties of the algorithms when considering the continuity of mean normal stress and the continuity of mean total normal stress for a wide range of flow regimes. Finally, examples in the physiological regime are shown to evaluate the importance, or not, of considering the continuity of mean total normal stress in hemodynamics simulations.
\end{abstract}

(ㄷ) 2013 Elsevier Inc. All rights reserved.

\section{Introduction}

The use of coupled dimensionally-heterogeneous models in hemodynamics simulations has been disseminated through the last years. In particular, when modeling arterial blood flow, this amounts to put together three-dimensional (3-D) fluidstructure interaction (FSI) models and one-dimensional (1-D), or lumped parameters, models. This allows to consider different levels of complexity at different places of the cardiovascular system or also to impose correct interaction between local 3-D dynamics and the global circulation. In this context several applications have been addressed, e.g., [1-8].

One of the most popular ways to couple 1-D and 3-D FSI models is given by imposing the conservation of mass and the continuity of mean normal stress (i.e., the normal component of the traction vector in a 3-D model) at coupling interfaces. This is supported by arguments based on both the strong formulation [9] and the variational formulation [10] of the interface problem.

Recently, in [11] the concept of energetic consistency between 1-D and 3-D FSI models has been introduced. More precisely, the authors show that the imposition of the conservation of mass and the continuity of mean normal stress at the

\footnotetext{
* Corresponding author. Tel.: +41 216932733.

E-mail addresses: pjblanco@lncc.br (P.J. Blanco), simone.deparis@epfl.ch (S. Deparis), cristiano.malossi@epfl.ch (A. Cristiano I. Malossi).
} 
coupling interfaces does not guarantee that the energy of the coupled system is preserved, i.e., it is not possible to write an energy estimate. The same authors state that, as a drawback, instabilities could occur at the interface between the models, although neither in their work, nor in the aforementioned works numerical instabilities have been reported. More generally, instabilities might arise in particular situations such as (i) in presence of substantial in-plane velocity fields near the boundary interfaces, which can be addressed by adding a weak constraint on the inlet/outlet velocity profiles, as proposed in [12], or (ii) by an uncontrolled reversal flow at the outlet boundaries, which might be avoided by adding an arbitrary traction to the outflow face whenever the reversal of the flow is detected, as proposed in [13].

With the aim to preserve the total energy of the problem, in [14,15] as well as in [11], an energetic preserving set of interface equations has been proposed, which is based on the conservation of mass and on the continuity of mean total normal stress. The main drawback of this set of interface equations is that it does not lead to classical type of boundary data for the connected models. More precisely, in the classical Navier-Stokes formulation the total normal stress cannot be imposed as a boundary condition. A possible workaround is to impose the flow rate on the 3-D coupling interfaces, as done in [11]. This is possible within the context of an iterative approach towards performing the coupling between models. Indeed, in [11] the authors use a Gauss-Seidel iterative procedure where the 3-D and the 1-D FSI models are fed with flow rate and total pressure boundary data, respectively. Nevertheless, the convergence properties are rather limited to the chosen numerical algorithm. In addition, the flexibility of choosing the boundary data to be applied on each model of the network is lost due to the need of prescribing a flow rate boundary condition on all the 3-D FSI coupling interfaces.

Another possible solution to meet the continuity of mean total normal stress, in presence of 3-D FSI models, is to change the Navier-Stokes formulation integrating by parts the convective term, as done in [14,15]. The resulting formulation is compatible with total stress boundary data. However, in a FSI context, the stress coupling condition at the interface between the fluid and the solid problem is also affected by the modifications in the 3-D Navier-Stokes formulation. Therefore, some additional changes in the FSI approach are required to match the new formulation. The solution to this issue is not discussed in the aforementioned works, as also pointed out in [16]. Moreover, from the software implementation viewpoint, any modification in the Navier-Stokes formulation, as well as in any FSI formulation, might be an issue when dealing with black box codes (e.g., commercial codes).

With the aim of preserving the appealing flexibility to impose the desired boundary data at each coupling interface of the network, being able, at the same time, to choose the set of interface equations without any constraint, we propose an algorithm compatible with a black box approach for the coupling of the heterogeneous models. An iterative strategy is adopted in order to decouple the 3-D and the 1-D problems. This is carried out following the ideas already proposed in [17,18]. However, the formulation presented in those works employed the conservation of mass and the continuity of mean normal stress at the interfaces. In this work we extend the interface problem in [18] to couple 3-D and 1-D FSI models by enforcing the conservation of mass and the continuity of mean total normal stress. The novelty is that the boundary data type is not forced by the local numerical scheme; the user can decide whether to impose a classical Neumann boundary condition (i.e., the normal stress) or a flow rate boundary data. The former is consistent with the FSI context and does not require modifications to the Navier-Stokes formulation, while the latter introduces a Lagrange multiplier in the system matrix. In addition, we perform a set of simulations to study the difference between the imposition of the conservation of mean normal stress and the conservation of mean total normal stress. These simulations range from benchmark examples to realistic applications in the modeling of the cardiovascular system.

This work is organized as follows. In Section 2 we briefly recall the 3-D and the 1-D FSI models from previous works. In Section 3 first we define the interface problem to couple heterogeneous models by imposing the mean normal stress and then we modify the formulation to account for the conservation of the mean total normal stress; furthermore, both cases are also extended to account for the continuity of the vessel area between two heterogeneous models. The numerical results are shown in Section 4, while the final remarks are outlined in Section 5.

\section{Fluid-structure interaction models}

In this section we briefly summarize the 3-D and the 1-D FSI models employed in this work. The governing equations are presented and references to the corresponding approximation techniques are given in due course.

\subsection{3-D FSI model}

Let $\Omega \subset \mathbb{R}^{3}$ with boundary $\partial \Omega$, where $\bar{\Omega}=\bar{\Omega}_{\mathrm{F}} \cup \bar{\Omega}_{\mathrm{S}}$, being $\Omega_{\mathrm{F}}$ and $\Omega_{\mathrm{S}}$ the fluid and solid domains, respectively. In addition, let $\Gamma_{\mathrm{I}}$ be the fluid-solid interface $\partial \Omega_{\mathrm{F}} \cap \partial \Omega_{\mathrm{S}}$. In the following we denote by the superscripts 0 and $t$ the reference and current configurations, respectively, of the fluid/solid domains and boundaries (see Fig. 1).

The field variables of the problem are the fluid velocity $\boldsymbol{u}_{\mathrm{F}}$, the fluid pressure $p_{\mathrm{F}}$, and the solid displacement $\boldsymbol{d}_{\mathrm{s}}$. The fluidstructure interface problem on the reference interface $\Gamma_{1}^{0}$ reads

$$
\begin{cases}\boldsymbol{u}_{\mathrm{F}} \circ \mathcal{M}^{t}-\frac{\partial \boldsymbol{d}_{\mathrm{S}}}{\partial t}=\mathbf{0} & \text { on } \Gamma_{\mathrm{I}}^{0} \times(0, T], \\ \sigma_{\mathrm{S}} \cdot \boldsymbol{n}_{\mathrm{S}}-J_{\mathrm{S}} \mathrm{G}_{\mathrm{S}}^{-\mathrm{T}}\left(\sigma_{\mathrm{F}} \circ \mathcal{M}^{t}\right) \cdot \boldsymbol{n}_{\mathrm{S}}=\mathbf{0} & \text { on } \Gamma_{\mathrm{I}}^{0} \times(0, T],\end{cases}
$$



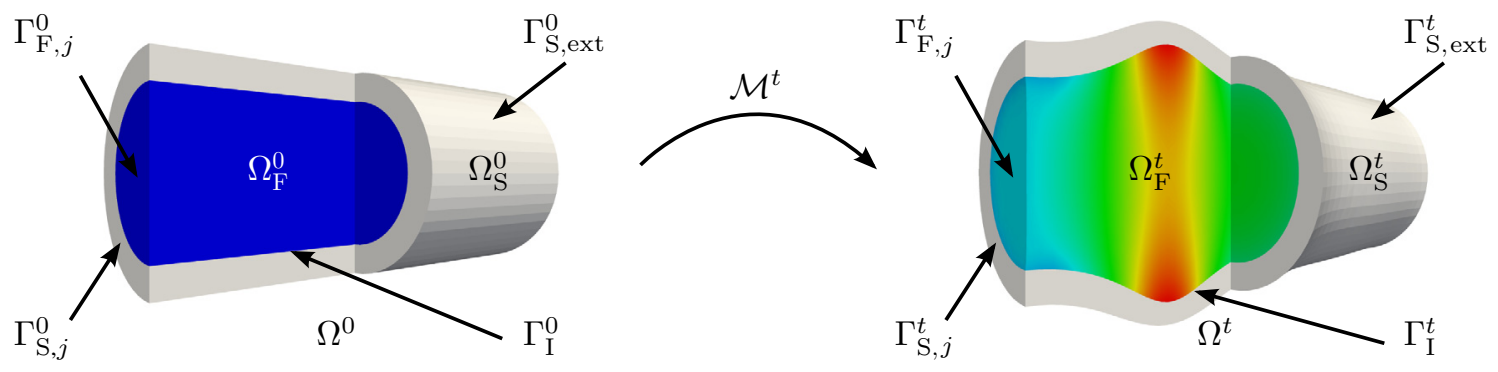

Fig. 1. Reference and current configurations with ALE mapping. The colors in the scheme refer to the computed pressure field. (For interpretation of the references to color in this figure legend, the reader is referred to the web version of this article.)

where $(0, T]$ is the time interval, $\boldsymbol{n}_{\mathrm{S}}$ the outgoing normal direction of the solid domain, and $\mathcal{M}^{t}$ the reference-to-current configuration map, in this context given by the chosen Arbitrary Lagrangian Eulerian (ALE) map, i.e.,

$$
\begin{aligned}
\mathcal{M}^{t}: & \Omega_{\mathrm{F}}^{0} \\
\boldsymbol{x}^{0} & \mapsto \Omega_{\mathrm{F}}^{t} \subset \mathcal{M}^{t}\left(\boldsymbol{x}^{0}\right)=\boldsymbol{x}^{0}+\boldsymbol{d}_{\mathrm{F}}\left(\boldsymbol{x}^{0}\right),
\end{aligned}
$$

such that $\Omega_{\mathrm{F}}^{t}=\mathcal{M}^{t}\left(\Omega_{\mathrm{F}}^{0}\right)$, with $\boldsymbol{x}^{0} \in \Omega_{\mathrm{F}}^{0}$ a fluid point in the reference configuration. In addition, $\mathrm{G}_{\mathrm{S}}=\mathrm{I}+\boldsymbol{\nabla} \boldsymbol{d}_{\mathrm{S}}$ is the solid deformation gradient, with $J_{\mathrm{S}}=\operatorname{det}\left(\mathrm{G}_{\mathrm{S}}\right)$, while $\sigma_{\mathrm{F}}$ and $\sigma_{\mathrm{S}}$ are the fluid and solid stress tensors, respectively.

The fluid problem consists of the incompressible Navier-Stokes equations written in the ALE formulation

$$
\begin{cases}\rho_{\mathrm{F}}\left(\left.\frac{\partial \boldsymbol{u}_{\mathrm{F}}}{\partial t}\right|_{\boldsymbol{x}^{0}}+\left(\left(\boldsymbol{u}_{\mathrm{F}}-\boldsymbol{w}_{\mathrm{F}}\right) \cdot \nabla\right) \boldsymbol{u}_{\mathrm{F}}\right)-\nabla \cdot \sigma_{\mathrm{F}}=\mathbf{0} & \text { in } \Omega_{\mathrm{F}}^{t} \times(0, T], \\ \nabla \cdot \boldsymbol{u}_{\mathrm{F}}=0 & \text { in } \Omega_{\mathrm{F}}^{t} \times(0, T], \\ \boldsymbol{u}_{\mathrm{F}}-\overline{\boldsymbol{u}}_{\mathrm{F}}=\mathbf{0} & \text { in } \Omega_{\mathrm{F}}^{t} \times\{0\},\end{cases}
$$

where $\rho_{\mathrm{F}}$ is the fluid density, $\sigma_{\mathrm{F}}=-p_{\mathrm{F}} \mathrm{I}+2 \mu_{\mathrm{F}} \epsilon_{\mathrm{F}}\left(\boldsymbol{u}_{\mathrm{F}}\right)$, I the identity matrix, $\epsilon_{\mathrm{F}}\left(\boldsymbol{u}_{\mathrm{F}}\right)$ the strain rate tensor, $\mu_{\mathrm{F}}$ the dynamic viscosity, $\boldsymbol{w}_{\mathrm{F}}$ the fluid domain velocity (defined by the ALE map), and $\overline{\boldsymbol{u}}_{\mathrm{F}}$ the initial fluid velocity field. Problem (1) is closed by inflow and outflow boundary conditions on $\Gamma_{\mathrm{F}, j}^{t} \subset \partial \Omega_{\mathrm{F}}^{t} \backslash \Gamma_{\mathrm{I}}^{t}, j=1, \ldots, n_{\mathrm{FS}}^{\Gamma}$. Note that since we are in the geometrical multiscale context, some of these boundaries are coupling interfaces with surrounding models.

The fluid domain displacement $\boldsymbol{d}_{\mathrm{F}}$ is computed by means of a geometrical problem as the harmonic extension of $\boldsymbol{d}_{\mathrm{s}}$ (given on $\Gamma_{\mathrm{I}}^{0}$, and which changes in time) to the interior of the fluid reference domain $\Omega_{\mathrm{F}}^{0}$, i.e.,

$$
\begin{cases}-\Delta \boldsymbol{d}_{\mathrm{F}}=\mathbf{0} & \text { in } \Omega_{\mathrm{F}}^{0}, \\ \boldsymbol{d}_{\mathrm{F}}-\boldsymbol{d}_{\mathrm{S}}=\mathbf{0} & \text { on } \Gamma_{\mathrm{I}}^{0}, \\ \nabla \boldsymbol{d}_{\mathrm{F}} \cdot \boldsymbol{n}_{\mathrm{F}}=\mathbf{0} & \text { on } \partial \Omega_{\mathrm{F}}^{0} \backslash \Gamma_{\mathrm{I}}^{0},\end{cases}
$$

being $\boldsymbol{n}_{\mathrm{F}}$ the outgoing normal direction of the fluid domain boundary.

The solid problem is described in a purely Lagrangian frame of reference. We consider a linear elastic isotropic St. VenantKirchhoff model to describe the material response

$$
\begin{cases}\rho_{\mathrm{S}} \frac{\partial^{2} \boldsymbol{d}_{\mathrm{S}}}{\partial t^{2}}-\nabla \cdot \sigma_{\mathrm{S}}=\mathbf{0} & \text { in } \Omega_{\mathrm{S}}^{0} \times(0, T] \\ \boldsymbol{d}_{\mathrm{S}}-\overline{\boldsymbol{d}}_{\mathrm{S}}=\mathbf{0} & \text { in } \Omega_{\mathrm{S}}^{0} \times\{0\} \\ \frac{\partial \boldsymbol{d}_{\mathrm{S}}}{\partial t}-\overline{\boldsymbol{v}}_{\mathrm{S}}=\mathbf{0} & \text { in } \Omega_{\mathrm{S}}^{0} \times\{0\}\end{cases}
$$

where $\rho_{\mathrm{S}}$ is the solid density, $\sigma_{\mathrm{S}}=\lambda_{\mathrm{S}} \operatorname{tr}\left(\epsilon_{\mathrm{S}}\left(\boldsymbol{d}_{\mathrm{S}}\right)\right) \mathrm{I}+2 \mu_{\mathrm{S}} \epsilon_{\mathrm{S}}\left(\boldsymbol{d}_{\mathrm{S}}\right), \epsilon_{\mathrm{S}}\left(\boldsymbol{d}_{\mathrm{S}}\right)$ the linear strain tensor, $\lambda_{\mathrm{S}}$ and $\mu_{\mathrm{S}}$ are the first and second Lamé parameters, respectively, $\overline{\boldsymbol{d}}_{\mathrm{S}}$ is the initial solid displacement, and $\overline{\boldsymbol{v}}_{\mathrm{S}}$ the initial solid velocity. Recall that the relations between $\lambda_{\mathrm{S}}$ and $\mu_{\mathrm{S}}$, and the Young's modulus $E_{\mathrm{S}}$ and the Poisson's ratio $v_{\mathrm{S}}$ are

$$
\lambda_{\mathrm{S}}=\frac{E_{\mathrm{S}} v_{\mathrm{S}}}{\left(1-2 v_{\mathrm{S}}\right)\left(1+v_{\mathrm{S}}\right)}, \quad \mu_{\mathrm{S}}=\frac{E_{\mathrm{S}}}{2\left(1+v_{\mathrm{S}}\right)} .
$$

As with problem (1), also problem (2) requires proper boundary data on $\partial \Omega_{S}^{0} \backslash \Gamma_{I}^{0}$. More precisely, on the external wall $\Gamma_{\text {S.ext }}^{0}$ we apply either homogeneous Neumann or viscoelastic Robin boundary conditions; the latter accounts for the presence of the external tissues in hemodynamics simulations [19]. Regarding the inlet/outlet solid rings $\Gamma_{\mathrm{S}, j}^{0}, j=1, \ldots, n_{\mathrm{FS}}^{\Gamma}$, they can be either clamped, let free to deform, or scaled to match the area of surrounding models, as described in [18].

The FSI problem is solved by using a non-modular (monolithic) approach, whose details are given in [20,21]. The fluid problem is discretized in space by a $\mathbb{P} 1-\mathbb{P} 1$ finite element method, stabilized by an interior penalty technique [22]. The solid and the geometric problems are discretized in space by $\mathbb{P} 1$ finite elements. Regarding time discretizations for the incom- 
pressible Navier-Stokes equations on moving domains we use a first order Euler scheme, while for the structural problem, we use an explicit second order mid-point scheme. The time interval $[0, T]$ is split into subintervals $\left[t^{n}, t^{n+1}\right], n=0,1,2, \ldots$, such that $t^{n}=n \Delta t, \Delta t$ being the time step. The fluid and solid problems are coupled by using the geometric convective explicit time discretization, i.e., the fluid problem is linearized by considering explicit the fluid domain displacement and the convective term. This choice allows to split the solution of the geometric part (the harmonic extension) from the fluid-solid one, leading to two smaller linear systems and a significant reduction of the computational cost. For more details on the 3-D FSI problem see $[20,21]$.

\subsection{1-D FSI model}

The 1-D FSI model can be derived from the incompressible Navier-Stokes equations by presuming an axisymmetric tubular domain and by introducing hypotheses related to the behavior of the quantities across the cross sectional area and to the flow regime [23] (see Fig. 2). The resulting governing equations for continuity of mass and momentum are

$$
\begin{cases}\frac{\partial A}{\partial t}+\frac{\partial Q}{\partial z}=0 & \text { in }(0, L) \times(0, T], \\ \frac{\partial Q}{\partial t}+\frac{\partial}{\partial z}\left(\alpha_{\mathrm{F}} \frac{Q^{2}}{A}\right)+\frac{A}{\rho_{\mathrm{F}}} \frac{\partial P}{\partial z}+\kappa_{\mathrm{F}} \frac{Q}{A}=0 & \text { in }(0, L) \times(0, T], \\ A-\bar{A}=0, \quad Q-\bar{Q}=0 & \text { in }(0, L) \times\{0\},\end{cases}
$$

where $A$ and $\bar{A}$ are the current and initial cross-sectional areas, respectively, $Q$ and $\bar{Q}$ are the current and initial volumetric flow rates, respectively, and $P$ is the average pressure. In addition, $\alpha_{\mathrm{F}}$ and $\kappa_{\mathrm{F}}$ are the Coriolis and friction coefficients, respectively, whose definitions are given, e.g., in [17].

The fluid problem (3) is coupled with the 1-D structural model through the pressure-area constitutive relation. As in $[17,18]$ we take into account the elastic and viscoelastic responses of the vessel wall, such that

$$
P=\psi(A)=P_{\text {ext }}+\beta_{S}\left(\sqrt{\frac{A}{A^{0}}}-1\right)+\gamma_{S}\left(\frac{1}{A \sqrt{A}} \frac{\partial A}{\partial t}\right) \quad \text { in }(0, L) \times(0, T],
$$

being $P_{\text {ext }}$ a reference external pressure, $A^{0}$ the value of the vessel area corresponding to a steady state with $P=P_{\text {ext }}$, and

$$
\beta_{\mathrm{S}}=\sqrt{\frac{\pi}{A^{0}}} \frac{h_{\mathrm{S}} E_{\mathrm{S}}}{1-v_{\mathrm{S}}^{2}}, \quad \gamma_{\mathrm{S}}=\frac{T_{\mathrm{S}} \tan \phi_{\mathrm{S}}}{4 \sqrt{\pi}} \frac{h_{\mathrm{S}} E_{\mathrm{S}}}{1-v_{\mathrm{S}}^{2}},
$$

where $h_{\mathrm{S}}$ is the wall thickness, $T_{\mathrm{S}}$ the wave characteristic time, and $\phi_{\mathrm{S}}$ the viscoelastic angle, which represents the phase difference between flow rate and pressure waves in the frequency domain.

The 1-D FSI problem is closed by proper boundary conditions on both sides of the 1-D domain. As in the 3-D case, these conditions can be either given data or quantities determined by solving the interface problem with the surrounding models. Finally, the 1-D FSI problem is solved by using an operator splitting technique based on an explicit second order TaylorGalerkin discretization, where the solution algorithm is split into two steps where the first one computes the result of a purely elastic problem, while the second one provides a viscoelastic correction. The spatial discretization is accomplished using $\mathbb{P} 1$ finite elements. For more details see [17].

\section{Interface problem}

In Section 2 we have introduced two FSI models characterized by different geometrical dimensions and governed by different kinds of partial differential equations, which in turn require different numerical methods to compute the solution. As

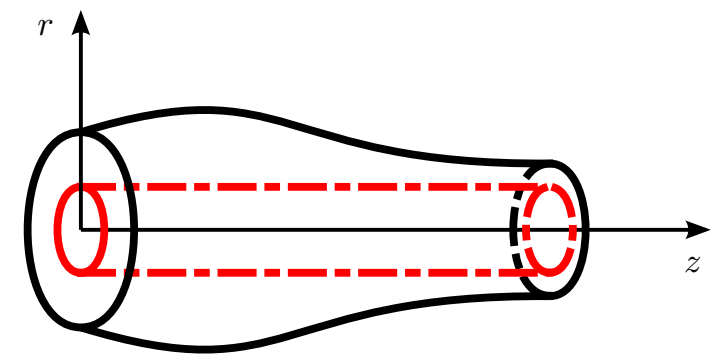

(a)

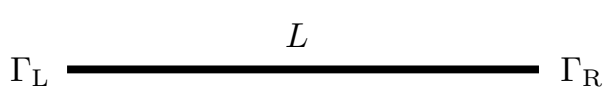

(b)

Fig. 2. Scheme of the 1-D FSI model. (a) The vessel is assumed to be a straight cylinder with a circular cross-section: the red (dashed) and black (solid) lines indicate the reference and current configurations, respectively. (b) The resulting 1-D model is a straight line with two boundary interfaces. (For interpretation of the references to color in this figure legend, the reader is referred to the web version of this article.) 
in previous works dealing with heterogeneous models, the coupling between them is set by imposing the conservation of averaged/integrated quantities over the interfaces, removing any dependency from the geometrical nature and the mathematical formulation of each model. More precisely, let us consider a general network of heterogeneous models connected by $\mathcal{C}$ coupling nodes. Following [24], at each $c$-th coupling node we impose a set of interface equations for the fluid part of the problem (see Fig. 3). Being $\chi_{c}$ the local vector of unknowns, the corresponding local residual form at the $c$-th coupling node is

$$
\boldsymbol{\mathcal { R }}_{c}\left(\boldsymbol{\chi}_{c}, \cdot\right)=\mathbf{0}
$$

where $c=1, \ldots, \mathcal{C}$, while the dot in the round parentheses expresses a possible dependence of the local residual on non-local unknowns, i.e., quantities defined at other coupling nodes of the underlying models.

Let $\boldsymbol{\chi}_{\mathcal{G}}=\left(\boldsymbol{\chi}_{1}^{\top}, \boldsymbol{\chi}_{2}^{\top}, \ldots, \boldsymbol{\chi}_{\mathcal{C}}^{\top}\right)^{\top}$ be the global vector of unknowns; more generally, in the following we use the subscript $\mathcal{G}$ to refer to quantities of the global interface problem. The solution of the global coupled problem is addressed by using a nonlinear Richardson strategy

$$
\chi_{\mathcal{G}}^{k+1}=\chi_{\mathcal{G}}^{k}+\delta \chi_{\mathcal{G}}^{k}
$$

until convergence within a suitable tolerance has been achieved. Being

$$
\boldsymbol{\mathcal { R }}_{\mathcal{G}}\left(\boldsymbol{\chi}_{\mathcal{G}}\right)=\left(\boldsymbol{\mathcal { R }}_{1}\left(\boldsymbol{\chi}_{1}, \cdot\right)^{\top}, \boldsymbol{\mathcal { R }}_{2}\left(\boldsymbol{\chi}_{2}, \cdot\right)^{\top}, \ldots, \boldsymbol{\mathcal { R }}_{\mathcal{C}}\left(\boldsymbol{\chi}_{\mathcal{C}}, \cdot\right)^{\top}\right)^{\top},
$$

the global residuals vector of the interface problem, the update $\delta \boldsymbol{\chi}_{\mathcal{G}}^{k}$ is computed by using either a Newton or an inexactNewton method by solving

$$
\mathcal{J}_{\mathcal{G}}\left(\boldsymbol{\chi}_{\mathcal{G}}^{k}\right) \delta \chi_{\mathcal{G}}^{k}=-\mathcal{R}_{\mathcal{G}}\left(\boldsymbol{\chi}_{\mathcal{G}}^{k}\right)
$$

In case the Newton method is used to solve (5), the Jacobian matrix of the global interface problem is given by the derivative of the residuals vector with respect to the coupling unknowns, i.e.,

$$
\mathcal{J}_{\mathcal{G}}\left(\boldsymbol{\chi}_{\mathcal{G}}\right)=\frac{\partial \mathcal{R}_{\mathcal{G}}\left(\boldsymbol{\chi}_{\mathcal{G}}\right)}{\partial \boldsymbol{\chi}_{\mathcal{G}}}
$$

In addition, in view of the results obtained in $[18,25,26]$ we also consider the Broyden method for the update at each nonlinear Richardson iteration, instead of recomputing the exact Jacobian matrix. In [24] other simpler schemes, i.e., the fixedpoint and the Aitken methods, have been tested over different benchmark examples leading to poorly convergent or even divergent results; for this reason these methods have not been considered in the current work.

In the following sections we detail the expression of the coupling equations behind (4). In particular, first we recall from [18] the set of equations for the imposition of the conservation of mass and the continuity of the mean normal stress. Then, we reformulate the interface problem in order to substitute the continuity of mean normal stress by the continuity of mean total normal stress. Finally, we extend both approaches to account for the continuity of the vessel area.

Before proceeding we remark that all the methodology presented here is general and holds not only for the two models presented in Section 2, but also for any other FSI formulation characterized, for example, by a nonlinear structure or higher order space and time discretizations. Anyway, regarding the notation, note that each 3-D FSI model has an arbitrary number of coupling interfaces $n_{\mathrm{FS}}^{\Gamma}$, while the 1-D FSI model has trivially two coupling interfaces, as shown in Fig. 4.

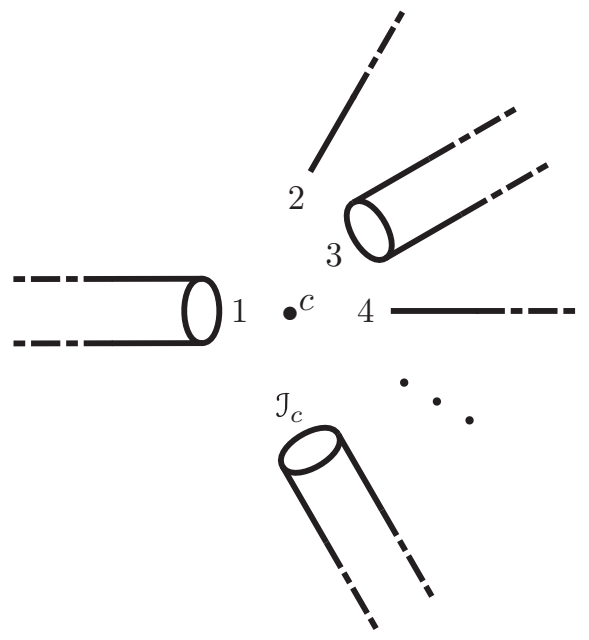

Fig. 3. Generic configuration for the $c$-th coupling between $\mathcal{I}_{c}$ dimensionally-heterogeneous models. 


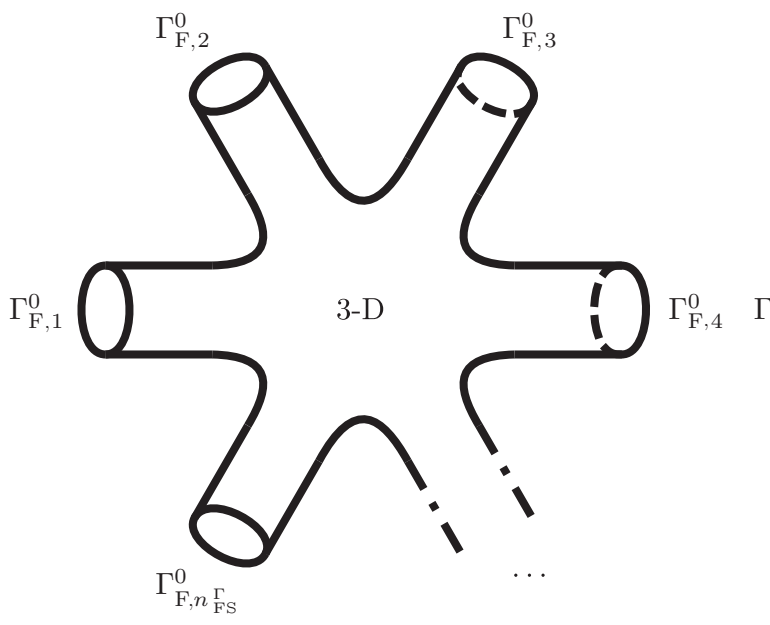

(a)

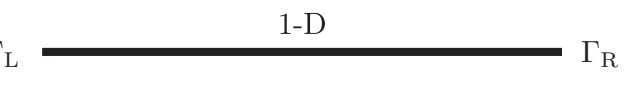

(b)

Fig. 4. Examples of 3-D (fluid domain $\Omega_{\mathrm{F}}^{0}$ ) and 1-D models with connections (coupling interfaces). (a) The 3-D model has $n_{\mathrm{FS}}^{\Gamma} \geqslant 1$ coupling interfaces. (b) The 1 -D model has two coupling interfaces.

\subsection{Continuity of mean normal stress}

A first set of interface equations can be obtained by ensuring the conservation of the mass and the continuity of the mean normal stress at the coupling nodes. This can be done by directly employing the boundary quantities of the fluid part of the problem, which are the volumetric flow rate $\mathcal{Q}$ and the averaged normal component of the traction vector $\mathcal{S}$, hereafter referred to as the coupling flow and the coupling stress, respectively. On the $j$-th coupling interface of the 3-D FSI model these quantities are computed as

$$
\mathcal{Q}_{j}^{3-\mathrm{D}}=\int_{\Gamma_{\mathrm{F}, j}^{t}} \boldsymbol{u}_{\mathrm{F}} \cdot \boldsymbol{n}_{\mathrm{F}} \mathrm{d} \Gamma, \quad \mathcal{S}_{j}^{3-\mathrm{D}}=\frac{1}{\left|\Gamma_{\mathrm{F}, j}^{t}\right|} \int_{\Gamma_{\mathrm{F}, j}^{t}}\left(\sigma_{\mathrm{F}} \cdot \boldsymbol{n}_{\mathrm{F}}\right) \cdot \boldsymbol{n}_{\mathrm{F}} \mathrm{d} \Gamma,
$$

where we assume that each boundary surface $\Gamma_{\mathrm{F}, j}^{t}, j=1, \ldots, n_{\mathrm{FS}}^{\Gamma}$, is planar and equipped with an outward normal vector $\boldsymbol{n}_{\mathrm{F}}$. In addition, since for modeling reasons we assume that at the boundary interfaces the flow is fully developed and orthogonal to the plane, the 3-D fluid problem is closed by imposing $\left(\sigma_{\mathrm{F}} \cdot \boldsymbol{n}_{\mathrm{F}}\right) \cdot \tau_{1 \mathrm{~F}}=0$ and $\left(\sigma_{\mathrm{F}} \cdot \boldsymbol{n}_{\mathrm{F}}\right) \cdot \tau_{2 \mathrm{~F}}=0$ on $\Gamma_{\mathrm{F}, j}^{t}, j=1, \ldots, n_{\mathrm{FS}}^{\Gamma}$, where $\tau_{1 \mathrm{~F}}$ and $\tau_{2 \mathrm{~F}}$ are the two tangential directions. In addition, we assume that the normal stress $\left(\sigma_{\mathrm{F}} \cdot \boldsymbol{n}_{\mathrm{F}}\right) \cdot \boldsymbol{n}_{\mathrm{F}}$ is constant over the boundaries, i.e., $\mathcal{S}_{j}^{3-\mathrm{D}}=\left(\sigma_{\mathrm{F}} \cdot \boldsymbol{n}_{\mathrm{F}}\right) \cdot \boldsymbol{n}_{\mathrm{F}}$. Regarding the two coupling interfaces of the 1-D FSI model we have

$$
\begin{array}{lll}
\mathcal{Q}_{\mathrm{L}}^{1-\mathrm{D}}=-Q_{\mathrm{L}}, & \mathcal{S}_{\mathrm{L}}^{1-\mathrm{D}}=-P_{\mathrm{L}}, & \text { on } \Gamma_{\mathrm{L}}, \\
\mathcal{Q}_{\mathrm{R}}^{1-\mathrm{D}}=Q_{\mathrm{R}}, & \mathcal{S}_{\mathrm{R}}^{1-\mathrm{D}}=-P_{\mathrm{R}}, & \text { on } \Gamma_{\mathrm{R}} .
\end{array}
$$

where the subscripts $\mathrm{L}$ and $\mathrm{R}$ stand for left and right quantities, respectively.

\subsubsection{Interface equations}

We denote by $\mathcal{L}^{\mathcal{S}}$ the set of coupling nodes where the continuity of mean normal stress is imposed; following [18] for $c \in \mathcal{L}^{\mathcal{S}}$ the interface equations read

$$
\left\{\begin{array}{l}
\sum_{i=1}^{\mathcal{I}_{c}} \mathcal{Q}_{c, i}=0, \\
\mathcal{S}_{c, 1}-\mathcal{S}_{c, i}=0, \quad i=2, \ldots, \mathcal{I}_{c},
\end{array}\right.
$$

where $\mathcal{I}_{c}$ is the local number of connected interfaces, $c=1, \ldots, \mathcal{C}$. The set of Eqs. (7), together with the nonlinear Richardson strategy used to solve the interface problem, are independent from the boundary data type to be imposed at each coupling interface of the different models. In other words, we can set up each subproblem with different combinations of boundary data over the coupling interfaces. Among the several possible combinations, in [24, Section 2.5] three significant cases are addressed. Here we generalize that approach writing a single residual form that holds in all the possible cases, i.e., 


$$
\mathcal{R}_{c}\left(\boldsymbol{\chi}_{c}, \cdot\right)=\left(\begin{array}{c}
\sum_{i=1}^{n_{c}^{\mathcal{Q}}} \mathcal{Q}_{c, i}+\sum_{i=n_{c}^{\mathcal{Q}}+1}^{\mathcal{I}_{c}} \mathcal{Q}_{c, i}\left(\mathcal{S}_{c}, \cdot\right) \\
\mathcal{S}_{c, 1}\left(\mathcal{Q}_{c, 1}, \cdot\right)-\mathcal{S}_{c} \\
\mathcal{S}_{c, 2}\left(\mathcal{Q}_{c, 2}, \cdot\right)-\mathcal{S}_{c} \\
\vdots \\
\mathcal{S}_{c, n_{c}^{\mathcal{Q}}}\left(\mathcal{Q}_{c, n_{c}^{\mathcal{Q}}}, \cdot\right)-\mathcal{S}_{c}
\end{array}\right), \quad \text { for } c \in \mathcal{L}^{\mathcal{S}},
$$

being $\mathcal{Q}_{c, i}(\cdot, \cdot)$ and $\mathcal{S}_{c, i}(\cdot, \cdot)$ two boundary operators that given a set of boundary conditions return the coupling flow and the coupling stress, respectively, at the $i$-th coupling interface of the $c$-th coupling node, while $n_{c}^{\mathcal{Q}}$ is the number of interfaces where a flow rate boundary datum is imposed, $0 \leqslant n_{c}^{\mathcal{Q}} \leqslant \mathcal{I}_{c}$; more precisely, we use the convention that among the $\mathcal{I}_{c}$ local coupling interfaces, the first $n_{c}^{\mathcal{Q}}$ take coupling flow boundary data, while the last $\mathcal{I}_{c}-n_{c}^{\mathcal{Q}}$ get coupling stress boundary data. In view of this, the size $\mathcal{L}_{c}$ of the unknowns vector $\chi_{c}$ is equal to $n_{c}^{\mathcal{Q}}+1$, such that

$$
\boldsymbol{\chi}_{c}=\left(\mathcal{Q}_{c, 1}, \mathcal{Q}_{c, 2}, \ldots, \mathcal{Q}_{c, n_{c}^{\mathcal{Q}}}, \mathcal{S}_{c}\right)^{\top}, \quad \text { for } c \in \mathcal{L}^{\mathcal{S}}
$$

In particular, if coupling stress boundary data are imposed on all the interfaces (i.e., $n_{c}^{\mathcal{Q}}=0$ ), the local node has just one associated unknown (the local coupling stress $\mathcal{S}_{c}$ ), while, in the dual case (coupling flow boundary data imposed on all the interfaces, i.e., $n_{c}^{\mathcal{Q}}=\mathcal{I}_{c}$ ), the local number of unknowns for that node is $\mathcal{I}_{c}+1$. Regarding the other terms in (8), we remark that the boundary operators $\mathcal{Q}_{c, i}\left(\mathcal{S}_{c}, \cdot\right)$ and $\mathcal{S}_{c, i}\left(\mathcal{Q}_{c, i}, \cdot\right)$ are global, as they may depend also on non-local coupling quantities, i.e., unknowns introduced at other coupling nodes; again, this dependence is indicated by the dot in the round parentheses.

The first equation in (8) ensures the conservation of mass at the $c$-th node. In case $n_{c}^{\mathcal{Q}}=0$, the continuity of the mean normal stress is satisfied implicitly by the fact that the same coupling stress $\mathcal{S}_{c}$ (which is the only local unknown, i.e., $\chi_{c}=\mathcal{S}_{c}$ ) is imposed on all the interfaces of the $c$-th node. In case $n_{c}^{\mathcal{Q}}>0$, on the interfaces where flow rate boundary data are imposed, the continuity of the mean normal stress is enforced explicitly through a relation of the form: $\mathcal{S}_{c, i}\left(\mathcal{Q}_{c, i}, \cdot\right)-\mathcal{S}_{c}=0, i=1, \ldots, n_{c}^{\mathcal{Q}}$.

Remark 1. In case $n_{\mathcal{C}}^{\mathcal{Q}}=\mathcal{I}_{c}$, i.e., coupling flow imposed on all the interfaces, the residual form can be reduced to have just $\mathcal{I}_{c}$ unknowns:

$$
\mathcal{R}_{c}\left(\boldsymbol{\chi}_{c}, \cdot\right)=\left(\begin{array}{c}
\sum_{i=1}^{\mathcal{I}_{c}} \mathcal{Q}_{c, i} \\
\mathcal{S}_{c, 2}\left(\mathcal{Q}_{c, 2}, \cdot\right)-\mathcal{S}_{c, 1}\left(\mathcal{Q}_{c, 1}, \cdot\right) \\
\vdots \\
\mathcal{S}_{c, \mathcal{I}_{c}}\left(\mathcal{Q}_{c, \mathcal{I}_{c}}, \cdot\right)-\mathcal{S}_{c, 1}\left(\mathcal{Q}_{c, 1}, \cdot\right)
\end{array}\right), \quad \text { for } c \in \mathcal{L}^{\mathcal{S}}
$$

being

$$
\chi_{c}=\left(\mathcal{Q}_{c, 1}, \mathcal{Q}_{c, 2}, \ldots, \mathcal{Q}_{c, \mathcal{I}_{c}}\right)^{\top}, \quad \text { for } c \in \mathcal{L}^{\mathcal{S}},
$$

where the unknown $\mathcal{S}_{c}$ has been dropped since the continuity of mean normal stress is now enforced explicitly on all the interface through a relation of the form: $\mathcal{S}_{c, i}\left(\mathcal{Q}_{c, i}, \cdot\right)-\mathcal{S}_{c, 1}\left(\mathcal{Q}_{c, 1}, \cdot\right)=0, i=1, \ldots, \mathcal{I}_{c}$.

Remark 2. Note that, independently of the value of $n_{c}^{\mathcal{Q}}$ and, consequently, from the chosen repartition of boundaries where coupling flow and coupling stress boundary data are applied, the solution of the problem is the same. In fact, the general form of the local residuals vector is derived from the set of interface equations without any approximation. Nevertheless, in case of rigid wall models (and incompressible flow regimes), e.g., 3-D Navier-Stokes equations, the imposition of flow rate boundary data cannot be used everywhere, since the incompressibility constraint is not fulfilled in the iterative process that leads to the solution of the system. In this case, to guarantee the well-posedness of the subproblems, the imposition of the flow rate on all boundaries of the same model must be avoided.

\subsubsection{Jacobian entries computation}

To solve the interface problem with the Newton method we have to compute the partial derivatives of the boundary operators corresponding to the different Jacobian entries, which are subsequently used to fill the different blocks of Jacobian matrix (6). The assembling procedure of the Jacobian matrix and the detailed description of the diagonal and off-diagonal blocks is extensively described in [27, Chapter 3]. Here, we mainly focus on the computation of the Jacobian entries: besides the constant values, there are four possible entries types, i.e.,

$$
\frac{\partial \mathcal{Q}_{c_{1}, j_{1}}\left(\boldsymbol{\chi}_{c_{1}}, \cdot\right)}{\partial \mathcal{Q}_{c_{2}, j_{2}}}, \quad \frac{\partial \mathcal{Q}_{c_{1}, j_{1}}\left(\boldsymbol{\chi}_{c_{1}}, \cdot\right)}{\partial \mathcal{S}_{c_{2}, j_{2}}}, \quad \frac{\partial \mathcal{S}_{c_{1}, j_{1}}\left(\boldsymbol{\chi}_{c_{1}}, \cdot\right)}{\partial \mathcal{Q}_{c_{2}, j_{2}}}, \quad \frac{\partial \mathcal{S}_{c_{1}, j_{1}}\left(\boldsymbol{\chi}_{c_{1}}, \cdot\right)}{\partial \mathcal{S}_{c_{2}, j_{2}}},
$$


where, for the sake of simplicity, since the boundary interfaces of the other models are not relevant at this level, we refer to the model boundary interfaces numeration, i.e., $j=1, \ldots, n_{\mathrm{FS}}^{\Gamma}$ or $j=\Gamma_{\mathrm{L}}, \Gamma_{\mathrm{R}}$, rather than to the node boundary interfaces one, i.e., $i=1, \ldots, \mathcal{I}_{c}$. More precisely, the indices $j_{1}$ and $j_{2}$, which are associated to the coupling nodes $c_{1}$ and $c_{2}$, respectively, refer to two boundary interfaces of the same model. Note that in case $c_{1} \equiv c_{2}$ the two boundary interfaces coincide and the first and last entries in (9) are equal to one.

These four Jacobian entry types are computed either solving the tangent problem associated to the coupled models, or by finite difference approximations. For the two FSI models described in Section 2, a detailed description of these approaches is provided in $[17,18,27]$.

\subsection{Continuity of mean total normal stress}

The second set of interface equations we consider ensures the conservation of the mass and the continuity of the mean total normal stress at the coupling nodes. Let us define the averaged normal component of the total stress on the boundary interfaces as $\mathcal{T}$, hereafter referred to as the coupling total stress. This quantity can be computed as the sum of the coupling stress and the dynamic contribution associated to the kinetic energy of the flow, which is a model-dependent quantity. More precisely, on the $j$-th coupling interface of the 3-D FSI model it is given by

$$
\mathcal{T}_{j}^{3-\mathrm{D}}=\mathcal{S}_{j}^{3-\mathrm{D}}-\frac{1}{2} \rho_{\mathrm{F}} \frac{1}{\left|\Gamma_{\mathrm{F}, j}^{t}\right|} \int_{\Gamma_{\mathrm{F}, j}^{t}}\left(\boldsymbol{u}_{\mathrm{F}} \cdot \boldsymbol{n}_{\mathrm{F}}\right)^{2} \mathrm{~d} \Gamma,
$$

while on both sides of the 1-D FSI model it is computed as

$$
\begin{array}{ll}
\mathcal{T}_{\mathrm{L}}^{1-\mathrm{D}}=\mathcal{S}_{\mathrm{L}}^{1-\mathrm{D}}-\frac{1}{2} \rho_{\mathrm{F}} \alpha_{\mathrm{F}}\left(\frac{\mathcal{Q}_{\mathrm{L}}^{1-\mathrm{D}}}{A}\right)^{2}, & \text { on } \Gamma_{\mathrm{L}}, \\
\mathcal{T}_{\mathrm{R}}^{1-\mathrm{D}}=\mathcal{S}_{\mathrm{R}}^{1-\mathrm{D}}-\frac{1}{2} \rho_{\mathrm{F}} \alpha_{\mathrm{F}}\left(\frac{\mathcal{Q}_{\mathrm{R}}^{1-\mathrm{D}}}{A}\right)^{2}, & \text { on } \Gamma_{\mathrm{R}} .
\end{array}
$$

\subsubsection{Interface equations}

To impose the continuity of the mean total normal stress we replace the coupling stress equations in (7) with an equivalent set of equations for the coupling total stress. We denote by $\mathcal{L}^{\mathcal{T}}$ the set of coupling nodes where the continuity of mean total normal stress is imposed; for $c \in \mathcal{L}^{\mathcal{T}}$ the coupling conditions read

$$
\left\{\begin{array}{l}
\sum_{i=1}^{\mathcal{I}_{c}} \mathcal{Q}_{c, i}=0, \\
\mathcal{I}_{c, 1}-\mathcal{T}_{c, i}=0, \quad i=2, \ldots, \mathcal{I}_{c} .
\end{array}\right.
$$

As with (7), system (10) does not depend on the imposed boundary data at each coupling interface of the models. However, in view of the aforementioned drawbacks associated to the imposition of the coupling total stress on the coupling interfaces, we consider only flow rate and mean normal stress as possible boundary data to set up the interface problem. The resulting generalized residual form reads

$$
\mathcal{R}_{c}\left(\boldsymbol{\chi}_{c}, \cdot\right)=\left(\begin{array}{c}
\sum_{i=1}^{n_{c}^{\mathcal{Q}}} \mathcal{Q}_{c, i}+\sum_{i=n_{c}^{\mathcal{Q}}+1}^{\mathcal{I}_{c}} \mathcal{Q}_{c, i}\left(\mathcal{S}_{c, i}, \cdot\right) \\
\mathcal{T}_{c, 1}\left(\mathcal{Q}_{c, 1}, \cdot\right)-\mathcal{T}_{c} \\
\mathcal{T}_{c, 2}\left(\mathcal{Q}_{c, 2}, \cdot\right)-\mathcal{T}_{c} \\
\vdots \\
\mathcal{T}_{c, n_{c}^{\mathcal{Q}}}\left(\mathcal{Q}_{c, n_{c}^{\mathcal{Q}}}, \cdot\right)-\mathcal{T}_{c} \\
\mathcal{T}_{c, n_{c}^{\mathcal{Q}}+1}\left(\mathcal{S}_{c, n_{c}^{\mathcal{Q}}+1}, \cdot\right)-\mathcal{T}_{c} \\
\mathcal{T}_{c, n_{c}^{\mathcal{Q}}+2}\left(\mathcal{S}_{c, n_{c}^{\mathcal{Q}}+2}, \cdot\right)-\mathcal{T}_{c} \\
\vdots \\
\mathcal{T}_{c, \mathcal{I}_{c}}\left(\mathcal{S}_{c, \mathcal{I}_{c}}, \cdot\right)-\mathcal{T}_{c}
\end{array}\right), \quad \text { for } c \in \mathcal{L}^{\mathcal{T}}
$$

where $\mathcal{T}_{c, i}(\cdot, \cdot)$ is a boundary operator that given a set of boundary conditions returns the coupling total stress at the $i$-th coupling interface of the $c$-th coupling node. The size $\mathcal{L}_{c}$ of the unknowns vector $\chi_{c}$ does not depend on the chosen set of interface quantities and is always equal to $\mathcal{I}_{c}+1$, such that

$$
\boldsymbol{\chi}_{c}=\left(\mathcal{Q}_{c, 1}, \mathcal{Q}_{c, 2}, \ldots, \mathcal{Q}_{c, n_{c}^{\mathcal{Q}}}, \mathcal{S}_{c, n_{c}^{\mathcal{Q}}+1}, \mathcal{S}_{c, n_{c}^{\mathcal{Q}}+2}, \ldots, \mathcal{S}_{c, \mathcal{I}_{c}}, \mathcal{T}_{c}\right)^{\top}, \quad \text { for } c \in \mathcal{L}^{\mathcal{T}}
$$


Therefore, compared to the previous case, if coupling stress boundary data are imposed on all the interfaces (i.e., $n_{c}^{\mathcal{Q}}=0$ ), the local interface problem has $\mathcal{I}_{c}$ additional unknowns, while, in the dual case (coupling flow boundary data imposed on all the interfaces, i.e., $n_{c}^{\mathcal{Q}}=\mathcal{I}_{c}$ ), the local number of unknowns is the same as before.

As in the set of coupling conditions introduced in Section 3.1, the first equation in (11) ensures the conservation of mass. The other $\mathcal{I}_{c}$ equations enforce the continuity of the mean total normal stress through an explicit relation of the form: $\mathcal{T}_{c, i}(\cdot, \cdot)-\mathcal{T}_{c}=0, i=1, \ldots, \mathcal{I}_{c}$, where we remark that the first (local to that node) argument in the round parenthesis can be either a coupling flow or a coupling stress, depending on the type of boundary condition imposed on the $i$-th interface.

Remark 3. Similarly to what has been done in Remark 1 for the continuity of the mean normal stress, also in this case it is possible to reduce the size of the residual form when $n_{\mathcal{C}}^{\mathcal{Q}}=\mathcal{I}_{c}$, such that

$$
\mathcal{R}_{c}\left(\boldsymbol{\chi}_{c}, \cdot\right)=\left(\begin{array}{c}
\sum_{i=1}^{\mathcal{I}_{c}} \mathcal{Q}_{c, i} \\
\mathcal{T}_{c, 2}\left(\mathcal{Q}_{c, 2}, \cdot\right)-\mathcal{T}_{c, 1}\left(\mathcal{Q}_{c, 1}, \cdot\right) \\
\vdots \\
\mathcal{T}_{c, \mathcal{I}_{c}}\left(\mathcal{Q}_{c, \mathcal{I}_{c}}, \cdot\right)-\mathcal{T}_{c, 1}\left(\mathcal{Q}_{c, 1}, \cdot\right)
\end{array}\right), \quad \text { for } c \in \mathcal{L}^{\mathcal{T}}
$$

being

$$
\chi_{c}=\left(\mathcal{Q}_{c, 1}, \mathcal{Q}_{c, 2}, \ldots, \mathcal{Q}_{c, \mathcal{I}_{c}}\right)^{\top}, \quad \text { for } c \in \mathcal{L}^{\mathcal{T}} .
$$

Remark 4. In case the mean total normal stress is considered as boundary data for the models, the residual form can be rewritten as

$$
\mathcal{R}_{c}\left(\boldsymbol{\chi}_{c}, \cdot\right)=\left(\begin{array}{c}
\sum_{i=1}^{n_{c}^{\mathcal{Q}}} \mathcal{Q}_{c, i}+\sum_{i=n_{c}^{\mathcal{Q}}+1}^{\mathcal{I}_{c}} \mathcal{Q}_{c, i}\left(\mathcal{T}_{c}, \cdot\right) \\
\mathcal{T}_{c, 1}\left(\mathcal{Q}_{c, 1}, \cdot\right)-\mathcal{T}_{c} \\
\mathcal{T}_{c, 2}\left(\mathcal{Q}_{c, 2}, \cdot\right)-\mathcal{T}_{c} \\
\vdots \\
\mathcal{T}_{c, n_{c}^{\mathcal{Q}}\left(\mathcal{Q}_{c, n_{c}^{\mathcal{Q}}}, \cdot\right)-\mathcal{T}_{c}}
\end{array}\right), \quad \text { for } c \in \mathcal{L}^{\mathcal{T}}
$$

being

$$
\chi_{c}=\left(\mathcal{Q}_{c, 1}, \mathcal{Q}_{c, 2}, \ldots, \mathcal{Q}_{c, n_{c}^{\mathcal{Q}}}, \mathcal{T}_{c}\right)^{\top}, \quad \text { for } c \in \mathcal{L}^{\mathcal{T}} .
$$

The resulting residuals and unknowns vectors are similar to those described in Section 3.1; indeed, the only difference between the two cases is that the coupling stress unknown and operators are replaced with those corresponding to the coupling total stress.

\subsubsection{Jacobian entries computation}

As done in Section 3.1.2 for the continuity of the mean normal stress, here we provide some details about the computation of the Jacobian entries when imposing the continuity of the mean total normal stress. More precisely, deriving (11) with respect to the available coupling quantities, we get four different entry types, i.e.,

$$
\frac{\partial \mathcal{Q}_{c_{1}, j_{1}}\left(\boldsymbol{\chi}_{c_{1}}, \cdot\right)}{\partial \mathcal{Q}_{c_{2}, j_{2}}}, \quad \frac{\partial \mathcal{Q}_{c_{1}, j_{1}}\left(\boldsymbol{\chi}_{c_{1}}, \cdot\right)}{\partial \mathcal{S}_{c_{2}, j_{2}}}, \frac{\partial \mathcal{T}_{c_{1}, j_{1}}\left(\boldsymbol{\chi}_{c_{1}}, \cdot\right)}{\partial \mathcal{Q}_{c_{2}, j_{2}}}, \frac{\partial \mathcal{T}_{c_{1}, j_{1}}\left(\boldsymbol{\chi}_{c_{1}}, \cdot\right)}{\partial \mathcal{S}_{c_{2}, j_{2}}}
$$

where the indices follow the same conventions discussed in Section 3.1.2. The first two entries in (12) are computed as described in Section 3.1.2. Regarding the other two contributions, their computation depends on the type of FSI model to which they are associated. More precisely for the 3-D FSI model they are computed as

$$
\frac{\partial \mathcal{T}_{c_{1}, j_{1}}\left(\boldsymbol{\chi}_{c_{1}}, \cdot\right)}{\partial \mathcal{Q}_{c_{2}, j_{2}}}=\Theta^{\mathcal{Q}}+\mathcal{K}, \quad \frac{\partial \mathcal{T}_{c_{1}, j_{1}}\left(\chi_{c_{1}}, \cdot\right)}{\partial \mathcal{S}_{c_{2}, j_{2}}}=\Theta^{\mathcal{S}}+\mathcal{K}
$$

where

$$
\Theta^{\mathcal{Q}}=\left\{\begin{array}{ll}
\frac{\partial \mathcal{S}_{c_{1} j_{1}}\left(\boldsymbol{\chi}_{c_{1}}, \cdot\right)}{\partial \mathcal{Q}_{c_{2}, j_{2}}} & \text { if } j_{1} \in \Upsilon^{\mathcal{Q}}, \\
0 & \text { otherwise, }
\end{array} \quad \Theta^{\mathcal{S}}= \begin{cases}\frac{\partial \mathcal{S}_{c_{1}, j_{1}}\left(\boldsymbol{\chi}_{c_{1}}, \cdot\right)}{\partial \mathcal{S}_{c_{2}, j_{2}}} & \text { if } j_{1} \in \Upsilon^{\mathcal{Q}} \text { and } c_{1} \neq c_{2}, \\
1 & \text { if } j_{1} \in \Upsilon^{\mathcal{S}} \text { and } c_{1} \equiv c_{2}, \\
0 & \text { otherwise, }\end{cases}\right.
$$


being $\Upsilon^{\mathcal{Q}}$ and $\Upsilon^{\mathcal{S}}$ the local lists of indices associated to the boundary interfaces of $\Omega_{\mathrm{F}}^{t}$ where coupling flow and coupling stress boundary data, respectively, are applied, while the kinetic term derivative $\mathcal{K}$ is

$$
\begin{aligned}
\mathcal{K}= & \frac{1}{2} \rho_{\mathrm{F}} \frac{1}{\left|\Gamma_{\mathrm{F}, j_{1}}^{t}\right|^{2}}\left(\int_{\Gamma_{\mathrm{F} j_{1}}^{t}} \nabla_{\Gamma} \cdot \delta \boldsymbol{d}_{\mathrm{F}} \mathrm{d} \Gamma\right)\left(\int_{\Gamma_{\mathrm{F} j_{1}}^{t}}\left(\boldsymbol{u}_{\mathrm{F}} \cdot \boldsymbol{n}_{\mathrm{F}}\right)^{2} \mathrm{~d} \Gamma\right) \\
& -\frac{1}{2} \rho_{\mathrm{F}} \frac{1}{\left|\Gamma_{\mathrm{F}, j_{1}}^{t}\right|}\left(\int_{\Gamma_{\mathrm{F} j_{1}}^{t}} 2\left(\boldsymbol{u}_{\mathrm{F}} \cdot \boldsymbol{n}_{\mathrm{F}}\right)\left(\delta \boldsymbol{u}_{\mathrm{F}} \cdot \boldsymbol{n}_{\mathrm{F}}\right) \mathrm{d} \Gamma+\int_{\Gamma_{\mathrm{F} j_{1}}^{t}}\left(\nabla_{\Gamma} \cdot \delta \boldsymbol{d}_{\mathrm{F}}\right)\left(\boldsymbol{u}_{\mathrm{F}} \cdot \boldsymbol{n}_{\mathrm{F}}\right)^{2} \mathrm{~d} \Gamma\right),
\end{aligned}
$$

being $\nabla_{\Gamma}$. the surface divergence operator written on $\Gamma_{\mathrm{F}, j_{1}}^{t}$. We remark that all the quantities defined in (13) are obtained by solving the tangent problems of the corresponding models (see [18, Section 3.3.1]). Hence, the computational cost of the Jacobian entries for the two sets of Eqs. (7) and (10) is the same.

Regarding the 1-D FSI model we have

$$
\begin{aligned}
& \frac{\partial \mathcal{T}_{c_{1}, j_{1}}\left(\mathcal{Q}_{c_{1}, j_{1}}\right)}{\partial \mathcal{Q}_{c_{1}, j_{1}}}=\frac{\partial \mathcal{S}_{c_{1}, j_{1}}\left(\mathcal{Q}_{c_{1}, j_{1}}\right)}{\partial \mathcal{Q}_{c_{1}, j_{1}}}-\rho_{\mathrm{F}} \alpha_{\mathrm{F}}\left(\frac{\mathcal{Q}_{c_{1}, j_{1}}}{\mathcal{A}_{c_{1}, j_{1}}^{2}\left(\mathcal{Q}_{c_{1}, j_{1}}\right)}-\frac{\mathcal{Q}_{c_{1}, j_{1}}}{\mathcal{A}_{c_{1}, j_{1}}^{3}\left(\mathcal{Q}_{c_{1}, j_{1}}\right)} \frac{\partial \mathcal{A}_{c_{1}, j_{1}}\left(\mathcal{Q}_{c_{1}, j_{1}}\right)}{\partial \mathcal{Q}_{c_{1}, j_{1}}}\right), \\
& \frac{\partial \mathcal{T}_{c_{1}, j_{1}}\left(\mathcal{S}_{c_{1}, j_{1}}\right)}{\partial \mathcal{S}_{c_{1}, j_{1}}}=1-\rho_{\mathrm{F}} \alpha_{\mathrm{F}}\left(\frac{\mathcal{Q}_{c_{1}, j_{1}}\left(\mathcal{S}_{c_{1}, j_{1}}\right)}{\mathcal{A}_{c_{1}, j_{1}}^{2}\left(\mathcal{S}_{c_{1}, j_{1}}\right)} \frac{\partial \mathcal{Q}_{c_{1}, j_{1}}\left(\mathcal{S}_{c_{1}, j_{1}}\right)}{\partial \mathcal{S}_{c_{1}, j_{1}}}-\frac{\mathcal{Q}_{c_{1}, j_{1}}\left(\mathcal{S}_{c_{1}, j_{1}}\right)}{\mathcal{A}_{c_{1}, j_{1}}^{3}\left(\mathcal{S}_{c_{1}, j_{1}}\right)} \frac{\partial \mathcal{A}_{c_{1} j_{1}}\left(\mathcal{S}_{c_{1}, j_{1}}\right)}{\partial \mathcal{S}_{c_{1}, j_{1}}}\right),
\end{aligned}
$$

where we introduced the boundary operator $\mathcal{A}_{c}(\cdot)$ which expresses the dependence of the local area on the given boundary data at the $c$-th node. Note that in (14) we do not consider the case $c_{1} \neq c_{2}$, since due to the hyperbolic nature of the problem we can assume that a perturbation on the boundary condition imposed on one side of the 1-D segment does not have any effect on the other side during a sufficiently small period of time (for instance, a single time step [17, Section 3.3]).

The assembling procedure of the Jacobian matrix and the detailed description of the diagonal and off-diagonal blocks is extensively described in [27, Chapter 3] and is not reported here.

\subsection{Continuity of the vessel area}

In a geometrical multiscale setting, the interfaces of 3-D FSI models are generally connected with those of reduced models, such as 1-D FSI models. In this case, the boundary data for the 3-D solid ring can be automatically determined by writing an additional relation at the coupling interfaces of the two heterogeneous models to impose the continuity of the vessel area. This is done by using the strategy described in [18, Section 4.3], which is here extended to cover the case in which the continuity of the mean total normal stress is also enforced.

Remark 5. The value of the boundary area on the $j$-th solid ring of the 3-D FSI model is imposed through the following set of boundary conditions

$$
\begin{cases}\boldsymbol{d}_{\mathrm{S}} \cdot \boldsymbol{n}_{\mathrm{S}}=0 & \text { on } \Gamma_{\mathrm{I}}^{0} \cap \Gamma_{\mathrm{S}, \mathrm{j}}^{0} \times(0, T], \\ {\left[\boldsymbol{d}_{\mathrm{S}}-\Psi_{j}^{t}\left(\boldsymbol{x}^{0}-\boldsymbol{x}_{\mathrm{G}, j}^{0}\right)\right] \cdot \tau_{1 \mathrm{~S}}=0} & \text { on } \Gamma_{\mathrm{I}}^{0} \cap \Gamma_{\mathrm{S}, j}^{0} \times(0, T], \\ {\left[\boldsymbol{d}_{\mathrm{S}}-\Psi_{j}^{t}\left(\boldsymbol{x}^{0}-\boldsymbol{x}_{\mathrm{G}, j}^{0}\right)\right] \cdot \tau_{2 S}=0} & \text { on } \Gamma_{\mathrm{I}}^{0} \cap \Gamma_{\mathrm{S}, \mathrm{j}}^{0} \times(0, T],\end{cases}
$$

where the radial scale factor is defined as

$$
\Psi_{j}^{t}=\sqrt{\frac{A_{j}^{t}}{A_{j}^{0}}}-1,
$$

being $A_{j}^{0}$ and $\boldsymbol{x}_{\mathrm{G}, j}^{0}$ the reference area of the $j$-th coupling interface of the 3-D fluid problem and its geometric center, respectively. This approach preserves the original shape of each 3-D solid ring, whose size is scaled by the value of the given boundary area $A_{j}^{t}[18]$.

Let us define the area of the fluid section as $\mathcal{A}$, hereafter referred to as the coupling area. On the $j$-th coupling interface of the 3-D FSI model this quantity is computed as

$$
\mathcal{A}_{j}^{3-\mathrm{D}}=\left|\Gamma_{\mathrm{F}, j}^{t}\right| .
$$

Regarding the two coupling interfaces of the 1-D FSI model we have

$$
\begin{array}{ll}
\mathcal{A}_{\mathrm{L}}^{1-\mathrm{D}}=A_{\mathrm{L}}^{1-\mathrm{D}}, & \text { on } \Gamma_{\mathrm{L}}, \\
\mathcal{A}_{\mathrm{R}}^{1-\mathrm{D}}=A_{\mathrm{R}}^{1-\mathrm{D}}, & \text { on } \Gamma_{\mathrm{R}} .
\end{array}
$$


Table 1

Detailed form of the local residuals vector $\mathcal{R}_{c}\left(\boldsymbol{\chi}_{c}, \cdot\right)$ when the continuity of the vessel area is imposed between a 1-D and a 3-D FSI model. For each residuals vector the corresponding unknowns vector is given in Table 2 .

\begin{tabular}{|c|c|c|c|c|}
\hline$n_{\mathcal{C}}^{\mathcal{Q}}$ & $c \in \mathcal{L}^{\mathcal{S}} \cap \mathcal{L}^{\mathcal{A}}$ & & $c \in \mathcal{L}^{\mathcal{T}} \cap \mathcal{L}^{\mathcal{A}}$ & \\
\hline 0 & $\left(\begin{array}{l}\mathcal{Q}_{c, 1}^{1-\mathrm{D}}\left(\mathcal{S}_{c}, \cdot\right) \\
\mathcal{A}_{c, 1}^{1-\mathrm{D}}\left(\mathcal{S}_{c}, \cdot\right)\end{array}\right.$ & $\left.\begin{array}{l}+\mathcal{Q}_{c, 2}^{3-\mathrm{D}}\left(\mathcal{S}_{c}, \mathcal{A}_{c}, \cdot\right) \\
-\mathcal{A}_{c}\end{array}\right)$ & $\left(\begin{array}{r}\mathcal{Q}_{c, 1}^{1-\mathrm{D}}\left(\mathcal{S}_{c, 1}, \cdot\right) \\
\mathcal{T}_{c, 1}^{1-\mathrm{D}}\left(\mathcal{S}_{c, 1}, \cdot\right) \\
\mathcal{T}_{c, 2}^{3-\mathrm{D}}\left(\mathcal{S}_{c, 2}, \mathcal{A}_{c}, \cdot\right) \\
\mathcal{A}_{c, 1}^{1-\mathrm{D}}\left(\mathcal{S}_{c, 1}, \cdot\right)\end{array}\right.$ & $\left.\begin{array}{l}+\mathcal{Q}_{c, 2}^{3-\mathrm{D}}\left(\mathcal{S}_{c, 2}, \mathcal{A}_{c}, \cdot\right) \\
-\mathcal{T}_{c} \\
-\mathcal{T}_{c} \\
-\mathcal{A}_{c}\end{array}\right)$ \\
\hline 1 & $\left(\begin{array}{r}\mathcal{Q}_{c, 1} \\
\mathcal{S}_{c, 1}^{1-\mathrm{D}}\left(\mathcal{Q}_{c, 1}, \cdot\right) \\
\mathcal{A}_{c, 1}^{1-\mathrm{D}}\left(\mathcal{Q}_{c, 1}, \cdot\right)\end{array}\right.$ & $\left.\begin{array}{l}+\mathcal{Q}_{c, 2}^{3-\mathrm{D}}\left(\mathcal{S}_{c}, \mathcal{A}_{c}, \cdot\right) \\
-\mathcal{S}_{c} \\
-\mathcal{A}_{c}\end{array}\right)$ & $\begin{array}{r}\mathcal{Q}_{c, 1} \\
\mathcal{T}_{c, 1}^{1-\mathrm{D}}\left(\mathcal{Q}_{c, 1}, \cdot\right) \\
\mathcal{T}_{c, 2}^{3-\mathrm{D}}\left(\mathcal{S}_{c, 2}, \mathcal{A}_{c}, \cdot\right) \\
\mathcal{A}_{c, 1}^{1-\mathrm{D}}\left(\mathcal{Q}_{c, 1}, \cdot\right)\end{array}$ & $\left.\begin{array}{l}+\mathcal{Q}_{c, 2}^{3-\mathrm{D}}\left(\mathcal{S}_{c, 2}, \mathcal{A}_{c}, \cdot\right) \\
-\mathcal{T}_{c} \\
-\mathcal{T}_{c} \\
-\mathcal{A}_{c}\end{array}\right)$ \\
\hline 2 & $\begin{array}{r}\mathcal{Q}_{c, 1} \\
\mathcal{S}_{c, 1}^{1-\mathrm{D}}\left(\mathcal{Q}_{c, 1}, \cdot\right) \\
\mathcal{S}_{c, 2}^{3-\mathrm{D}}\left(\mathcal{Q}_{c, 2}, \mathcal{A}_{c}, \cdot\right) \\
\mathcal{A}_{c, 1}^{1-\mathrm{D}}\left(\mathcal{Q}_{c, 1}, \cdot\right)\end{array}$ & $\left.\begin{array}{ll}+ & \mathcal{Q}_{c, 2} \\
- & \mathcal{S}_{c} \\
- & \mathcal{S}_{c} \\
- & \mathcal{A}_{c}\end{array}\right)$ & 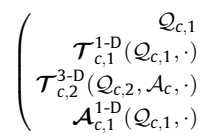 & $\left.\begin{array}{ll}+ & \mathcal{Q}_{c, 2} \\
- & \mathcal{T}_{c} \\
- & \mathcal{T}_{c} \\
- & \mathcal{A}_{c}\end{array}\right)$ \\
\hline
\end{tabular}

Table 2

Detailed form of the local unknowns vector $\chi_{c}$ when the continuity of the vessel area is imposed between a 1-D and a 3-D FSI model. For each unknowns vector the corresponding residuals vector is given in Table 1.

\begin{tabular}{lll}
\hline$n_{c}^{\mathcal{Q}}$ & $c \in \mathcal{L}^{\mathcal{S}} \cap \mathcal{L}^{\mathcal{A}}$ & $c \in \mathcal{L}^{\mathcal{T}} \cap \mathcal{L}^{\mathcal{A}}$ \\
\hline 0 & $\left(\mathcal{S}_{c}, \mathcal{A}_{c}\right)^{\top}$ & $\left(\mathcal{S}_{c, 1}, \mathcal{S}_{c, 2}, \mathcal{T}_{c}, \mathcal{A}_{c}\right)^{\top}$ \\
1 & $\left(\mathcal{Q}_{c, 1}, \mathcal{S}_{c}, \mathcal{A}_{c}\right)^{\top}$ & $\left(\mathcal{Q}_{c, 1}, \mathcal{S}_{c, 2}, \mathcal{T}_{c}, \mathcal{A}_{c}\right)^{\top}$ \\
2 & $\left(\mathcal{Q}_{c, 1}, \mathcal{Q}_{c, 2}, \mathcal{S}_{c}, \mathcal{A}_{c}\right)^{\top}$ & $\left(\mathcal{Q}_{c, 1}, \mathcal{Q}_{c, 2}, \mathcal{T}_{c}, \mathcal{A}_{c}\right)^{\top}$ \\
\hline
\end{tabular}

\subsubsection{Interface equations}

Let $\mathcal{L}^{\mathcal{A}}$ be the set of coupling nodes where the continuity of the area between the two heterogeneous FSI models is imposed. For $c \in \mathcal{L}^{\mathcal{S}} \cap \mathcal{L}^{\mathcal{A}}$ the set of Eqs. (7) becomes

$$
\left\{\begin{array}{l}
\mathcal{Q}_{c, 1}^{1-\mathrm{D}}+\mathcal{Q}_{c, 2}^{3-\mathrm{D}}=0, \\
\mathcal{S}_{c, 1}^{1-\mathrm{D}}-\mathcal{S}_{c, 2}^{3-\mathrm{D}}=0, \\
\mathcal{A}_{c, 1}^{1-\mathrm{D}}-\mathcal{A}_{c, 2}^{3-\mathrm{D}}=0,
\end{array}\right.
$$

while, for $c \in \mathcal{L}^{\mathcal{T}} \cap \mathcal{L}^{\mathcal{A}}$, the set of Eqs. (10) reads

$$
\left\{\begin{array}{l}
\mathcal{Q}_{c, 1}^{1-\mathrm{D}}+\mathcal{Q}_{c, 2}^{3-\mathrm{D}}=0, \\
\mathcal{T}_{c, 1}^{1-\mathrm{D}}-\mathcal{T}_{c, 2}^{3-\mathrm{D}}=0, \\
\mathcal{A}_{c, 1}^{1-\mathrm{D}}-\mathcal{A}_{c, 2}^{3-\mathrm{D}}=0 .
\end{array}\right.
$$

For the sake of clarity, in both cases the model to which each quantity belongs is indicated in the superscript. Indeed, we remark that (15) and (16) are written for the specific case of a 3-D FSI interface coupled with a single 1-D FSI model. In the case of a generalization to two or more 1-D models connected to the same 3-D FSI interface, the continuity of the area does not make sense, and for this reason we do not address this case. In addition, as described in [17], the 1-D FSI problem needs just one physical boundary condition on each side of the segment; by imposing either the coupling flow or the coupling stress, the value of the coupling area of the 1-D segment is already determined by the solution of the 1-D problem. In view of these considerations, for each coupling approach there are only three possible residual forms, depending on the value of $n_{c}^{\mathcal{Q}}$, as summarized in Table 1; the corresponding local vectors of unknowns are detailed in Table 2.

\subsubsection{Jacobian entries computation}

Deriving the residuals in Table 1 with respect to the available coupling quantities, we get eleven different entry types, i.e.,

$$
\begin{array}{llll}
\frac{\partial \mathcal{Q}_{c_{1}, j_{1}}\left(\boldsymbol{\chi}_{c_{1}}, \cdot\right)}{\partial \mathcal{Q}_{c_{2}, j_{2}}}, & \frac{\partial \mathcal{S}_{c_{1}, j_{1}}\left(\boldsymbol{\chi}_{c_{1}}, \cdot\right)}{\partial \mathcal{Q}_{c_{2}, j_{2}}}, & \frac{\partial \mathcal{T}_{c_{1}, j_{1}}\left(\boldsymbol{\chi}_{c_{1}}, \cdot\right)}{\partial \mathcal{Q}_{c_{2}, j_{2}}}, & \frac{\partial \mathcal{A}_{c_{1}, j_{1}}^{1-D}\left(\boldsymbol{\chi}_{c_{1}}, \cdot\right)}{\partial \mathcal{Q}_{c_{2}, j_{2}}}, \\
\frac{\partial \mathcal{Q}_{c_{1}, j_{1}}\left(\boldsymbol{\chi}_{c_{1}}, \cdot\right)}{\partial \mathcal{S}_{c_{2}, j_{2}}}, & \frac{\partial \mathcal{S}_{c_{1}, j_{1}}\left(\boldsymbol{\chi}_{c_{1}}, \cdot\right)}{\partial \mathcal{S}_{c_{2}, j_{2}}}, & \frac{\partial \mathcal{T}_{c_{1}, j_{1}}\left(\boldsymbol{\chi}_{c_{1}}, \cdot\right)}{\partial \mathcal{S}_{c_{2}, j_{2}}}, & \frac{\partial \mathcal{A}_{c_{1}, j_{1}}^{1-D}\left(\boldsymbol{\chi}_{c_{1}}, \cdot\right)}{\partial \mathcal{S}_{c_{2}, j_{2}}}, \\
\frac{\partial \mathcal{Q}_{c_{1}, j_{1}}^{3-D}\left(\boldsymbol{\chi}_{c_{1}}, \cdot\right)}{\partial \mathcal{A}_{c_{2}, j_{2}}}, & \frac{\partial \mathcal{S}_{c_{1}, j_{1}}^{3-D}\left(\boldsymbol{\chi}_{c_{1}}, \cdot\right)}{\partial \mathcal{A}_{c_{2}, j_{2}}}, & \frac{\partial \mathcal{T}_{c_{1}, j_{1}}^{3-D}\left(\boldsymbol{\chi}_{c_{1}}, \cdot\right)}{\partial \mathcal{A}_{c_{2}, j_{2}}},
\end{array}
$$


where the indices follow the same conventions used in Sections 3.1.2 and 3.2.2. The first three entries in the first two lines have already been discussed previously, while all the other entries appear here for the first time, since they are introduced by the presence of the coupling area (and its boundary operator) in the residual forms. More precisely, the last entries in the first two lines represent variations of the boundary area of the 1-D FSI model (as highlighted by the superscripts) due to variations of other coupling quantities (specifically, coupling flow and coupling stress), while the three entries in the last line represent variations of quantities defined in the 3-D FSI model due to a variation of the coupling area.

The computation of the last entries in the first two lines has already been addressed in [17,27], while that of the three entries in the last line can be achieved by using the procedure described in [18,27], where the mean total normal stress derivative is given by

$$
\frac{\partial \mathcal{T}_{c_{1}, j_{1}}\left(\boldsymbol{\chi}_{c_{1}}, \cdot\right)}{\partial \mathcal{A}_{c_{2}, j_{2}}}=\Theta^{\mathcal{A}}+\mathcal{K},
$$

with

$$
\Theta^{\mathcal{A}}= \begin{cases}\frac{\partial \mathcal{S}_{c_{1}, j_{1}}\left(\boldsymbol{\chi}_{c_{1}}, \cdot\right)}{\partial \mathcal{A}_{c_{2}, j_{2}}} & \text { if } j_{1} \in \Upsilon^{\mathcal{Q}} \\ 0 & \text { otherwise. }\end{cases}
$$

The assembling procedure of the Jacobian matrix and the detailed description of the diagonal and off-diagonal blocks is extensively described in [27, Chapter 3] and is not reported here.

\section{Results}

In this section we extend results presented in [18,19,24], where the continuity of the mean normal stress has been already extensively used, by using the continuity of the mean total normal stress to solve the same problems. The goal is to compare the results of the two approaches in terms of physics as well as from the point of view of the convergence properties of the analyzed algorithms. Moreover, we also consider situations in which the Jacobian matrix of the mean normal stress case is used to perform inexact-Newton iterations when enforcing the continuity of the mean total normal stress at the boundary interfaces. We refer to this strategy as the "approximated" case, where it is important to point out that the approximation is introduced at the level of the Jacobian matrix in the continuum problem. This approximation amounts to neglect the partial derivatives of the kinetic term and, evidently, does not affect the physics of the solution.

The layout of this section is the following: in Section 4.1 we consider a 3-D steady state benchmark case, with the aim to test the proposed methodology in a simple problem and for several Reynolds numbers. Then, in Section 4.2 we address the case of the coupling of two dimensionally-heterogeneous FSI pipes, comparing the amplitude of numerical reflections at the interface of the problem. Finally, in Section 4.3 we perform a numerical comparison on a realistic cardiovascular example, where a 3-D FSI model of the aorta is coupled with a network of 1-D FSI elements modeling the systemic circulation.

All the simulations presented here have been performed on one or more cluster nodes with two Intel ${ }^{\circledR}$ Xeon $^{\circledR}$ processors X5550 (quad core, $8 \mathrm{MB}$ cache, $2.66 \mathrm{GHz} \mathrm{CPU}$ ).

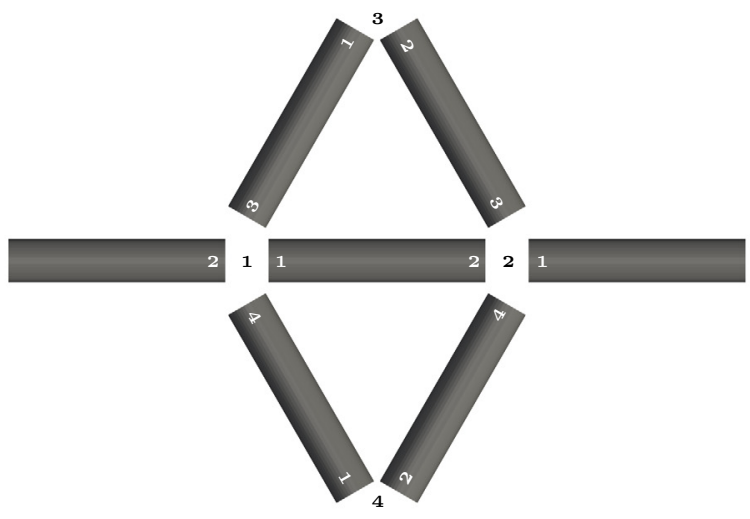

(a)

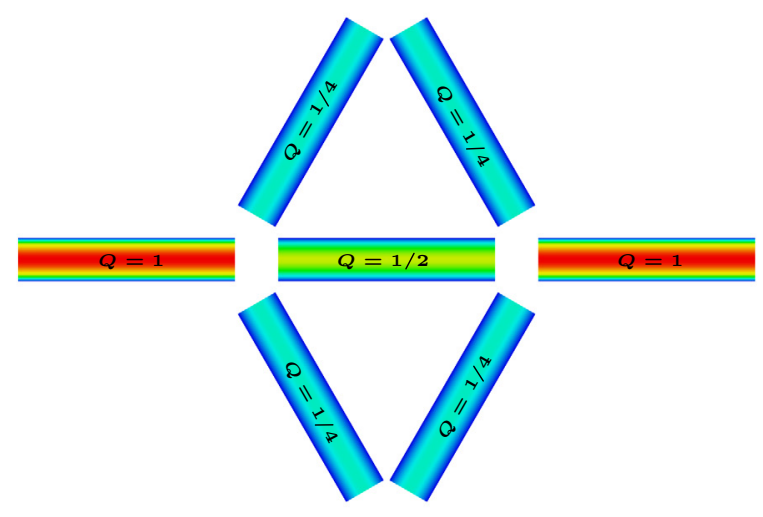

(b)

Fig. 5. Configuration and velocity magnitude of seven 3-D rigid wall pipes in a steady state regime. (a) Nodes and interfaces numeration: black and white numbers indicate the coupling nodes and the local numeration of the boundary interfaces, respectively. (b) Velocity magnitude: the color bar ranges from blue $(0)$ to red (400). (For interpretation of the references to color in this figure legend, the reader is referred to the web version of this article.) 


\subsection{Seven 3-D rigid wall pipes in a steady state regime}

The first example we consider is a 3-D rigid wall steady state problem (see [24, Section 4.1]), whose domain consists in a set of seven cylinders connected by four coupling interfaces, as shown in Fig. 5(a). All the cylinders have the same geometrical dimensions (diameter 0.08; length 0.4 ) and are discretized by the same mesh $(3,886,200$ tetrahedral elements and 670,464 vertices, with an average space discretizations of 0.00186). A unitary flow rate is imposed on the inflow on the leftmost side, while a homogeneous Neumann boundary condition is applied on the rightmost outflow. The value of the fluid density has

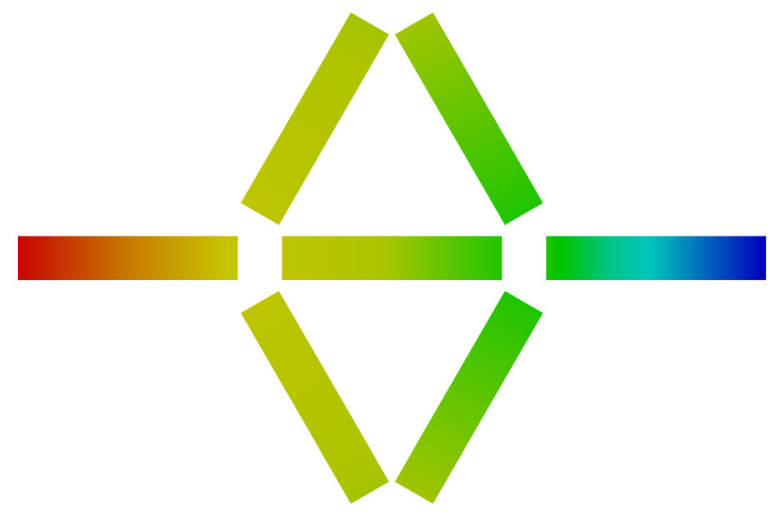

$c \in \mathcal{L}^{\mathcal{S}} \forall c ; \operatorname{Re}=1$; Color range: $0.0-1.6 \cdot 10^{7}$.

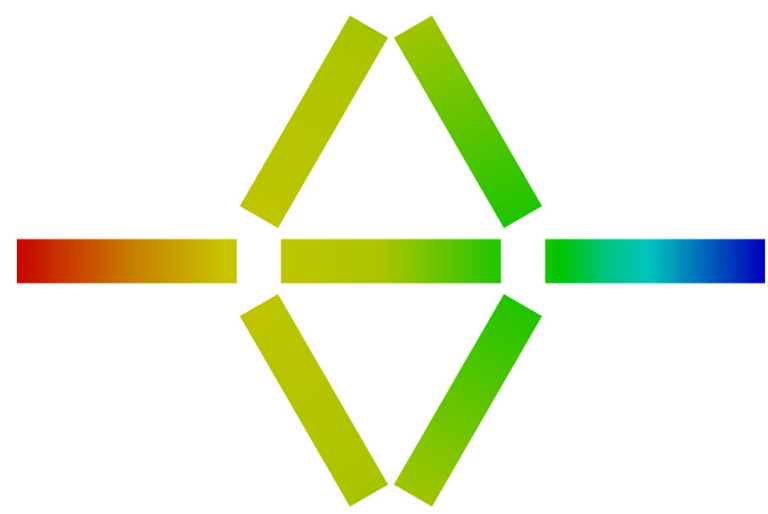

$c \in \mathcal{L}^{\mathcal{S}} \forall c ; \operatorname{Re}=1000$; Color range: $0.0-1.6 \cdot 10^{4}$.

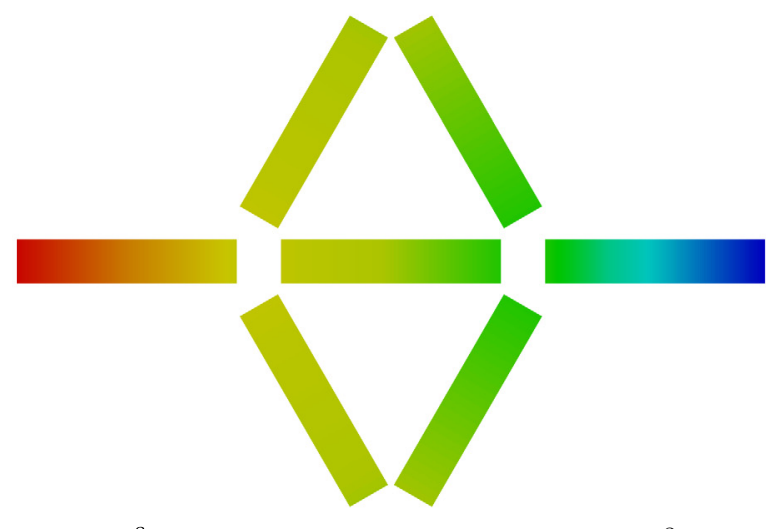

$c \in \mathcal{L}^{\mathcal{S}} \forall c ; \operatorname{Re}=10000$; Color range: $0.0-1.6 \cdot 10^{3}$.

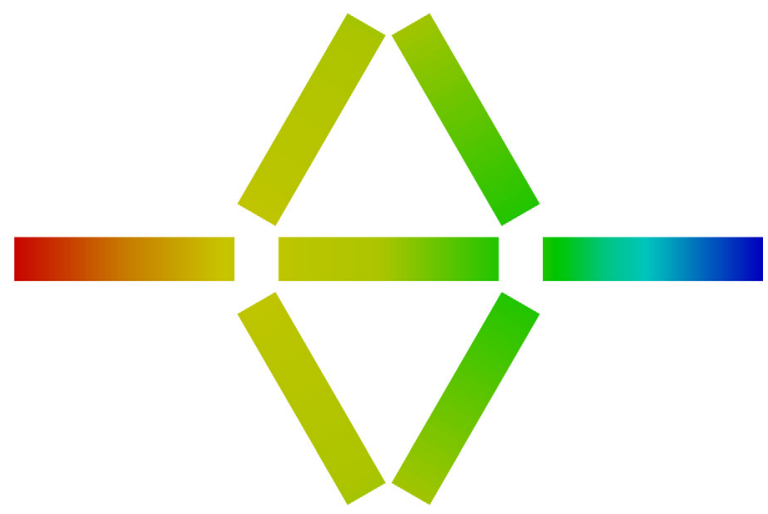

$c \in \mathcal{L}^{\mathcal{T}} \forall c ; \operatorname{Re}=1$; Color range: $0.0-1.6 \cdot 10^{7}$.

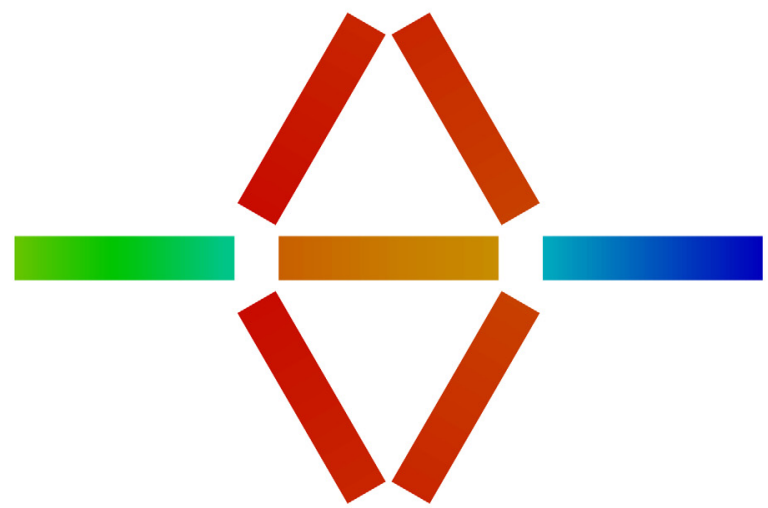

$c \in \mathcal{L}^{\mathcal{T}} \forall c ; \operatorname{Re}=1000 ;$ Color range: $0.0-3.5 \cdot 10^{4}$.

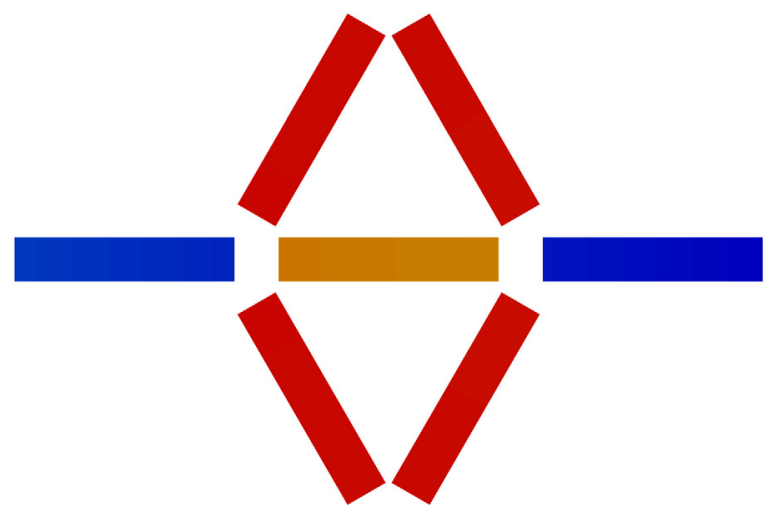

$c \in \mathcal{L}^{\mathcal{T}} \forall c ; \operatorname{Re}=10000$; Color range: $0.0-2.6 \cdot 10^{4}$.

Fig. 6. Pressure field of seven 3-D rigid wall pipes in a steady state regime, as a function of the chosen set of interface equations and of the Reynolds number. The color bar range (blue to red) is indicated in the caption of each subfigure. Note that in the case of continuity of the mean total normal stress the pressure is far from being continuous for high Reynolds numbers. (For interpretation of the references to color in this figure legend, the reader is referred to the web version of this article.) 
been set equal to one, while the dynamic viscosity is chosen in order to obtain the desired Reynolds number. All the quantities in the problem are dimensionless.

The resulting velocity magnitude and flow distribution are shown in Fig. 5(b). Note that the computed flow rate is exact up to the imposed tolerance for the linear solver. Regarding the pressure field and the stress boundary quantities, the solution changes as a function of the chosen set of interface equations and of the Reynolds number, as shown in Figs. 6, 7, Tables 3, and 4.

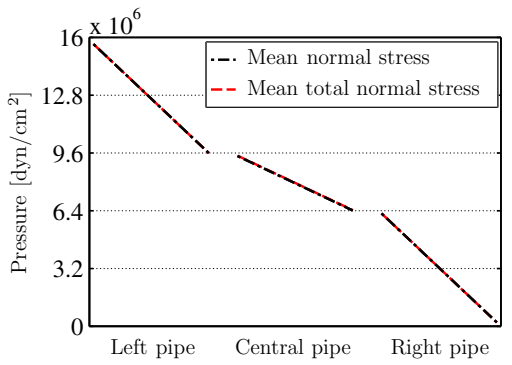

(a)

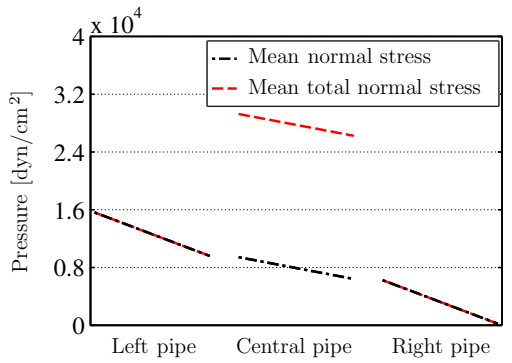

(b)

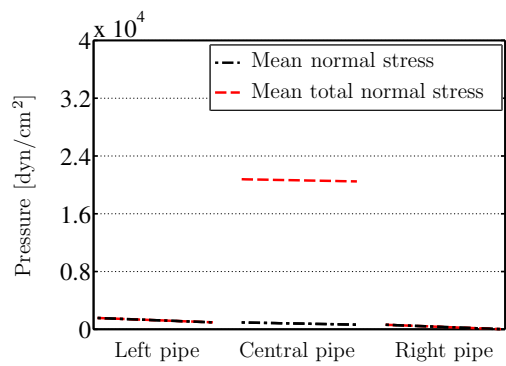

(c)

Fig. 7. Pressure field along the three central pipes of the seven cylinder topology in Fig. $5(\mathrm{a}) .(\mathrm{a}) \operatorname{Re}=1$. (b) $\operatorname{Re}=1000$. $(\mathrm{c}) \operatorname{Re}=10,000$.

Table 3

Coupling stress $\mathcal{S}_{c, i}$ at the coupling interfaces of the seven 3-D rigid wall pipes in a steady state regime, as a function of the Reynolds number (Re) and for different sets of interface equations.

\begin{tabular}{|c|c|c|c|c|c|c|c|}
\hline \multirow[t]{2}{*}{ c } & \multirow[t]{2}{*}{$i$} & \multicolumn{2}{|l|}{$\operatorname{Re}=1$} & \multicolumn{2}{|c|}{$\operatorname{Re}=1000$} & \multicolumn{2}{|c|}{$\mathrm{Re}=10,000$} \\
\hline & & $c \in \mathcal{L}^{\mathcal{S}}$ & $c \in \mathcal{L}^{\mathcal{T}}$ & $c \in \mathcal{L}^{\mathcal{S}}$ & $c \in \mathcal{L}^{\mathcal{T}}$ & $c \in \mathcal{L}^{\mathcal{S}}$ & $c \in \mathcal{L}^{\mathcal{T}}$ \\
\hline 1 & 1 & $-9,506,443$ & $-9,525,744$ & -9506 & $-29,333$ & -951 & $-20,783$ \\
\hline 1 & 2 & $-9,506,443$ & $-9,505,919$ & -9506 & -9506 & -951 & -951 \\
\hline 1 & 3,4 & $-9,506,443$ & $-9,530,701$ & -9506 & $-34,289$ & -951 & $-25,732$ \\
\hline 2 & 1 & $-6,337,759$ & $-6,337,368$ & -6338 & -6338 & -634 & -634 \\
\hline 2 & 2 & $-6,337,759$ & $-6,357,194$ & -6338 & $-26,164$ & -634 & $-20,467$ \\
\hline 2 & 3,4 & $-6,337,759$ & $-6,362,151$ & -6338 & $-31,120$ & -634 & $-25,415$ \\
\hline 3,4 & 1 & $-7,922,168$ & $-7,946,426$ & -7922 & $-32,704$ & -792 & $-25,573$ \\
\hline 3,4 & 2 & $-7,922,168$ & $-7,946,426$ & -7922 & $-32,704$ & -792 & $-25,573$ \\
\hline
\end{tabular}

Table 4

Coupling total stress $\mathcal{T}_{c, i}$ at the coupling interfaces of the seven 3-D rigid wall pipes in a steady state regime, as a function of the Reynolds number (Re) and for different sets of interface equations.

\begin{tabular}{|c|c|c|c|c|c|c|c|}
\hline \multirow[t]{2}{*}{ c } & \multirow[t]{2}{*}{$i$} & \multicolumn{2}{|l|}{$\operatorname{Re}=1$} & \multicolumn{2}{|l|}{$\operatorname{Re}=1000$} & \multicolumn{2}{|c|}{$\operatorname{Re}=10,000$} \\
\hline & & $c \in \mathcal{L}^{\mathcal{S}}$ & $c \in \mathcal{L}^{\mathcal{T}}$ & $c \in \mathcal{L}^{\mathcal{S}}$ & $c \in \mathcal{L}^{\mathcal{T}}$ & $c \in \mathcal{L}^{\mathcal{S}}$ & $c \in \mathcal{L}^{\mathcal{T}}$ \\
\hline 1 & 1 & $-9,512,974$ & $-9,532,353$ & $-16,115$ & $-35,941$ & -7559 & $-27,386$ \\
\hline 1 & 2 & $-9,533,223$ & $-9,532,353$ & $-35,940$ & $-35,941$ & $-27,384$ & $-27,386$ \\
\hline 1 & 3,4 & $-9,508,096$ & $-9,532,353$ & $-11,159$ & $-35,941$ & -2602 & $-27,386$ \\
\hline 2 & 1 & $-6,363,803$ & $-6,363,803$ & $-32,772$ & $-32,772$ & $-27,069$ & $-27,069$ \\
\hline 2 & 2 & $-6,344,424$ & $-6,363,803$ & $-12,946$ & $-32,772$ & -7242 & $-27,069$ \\
\hline 2 & 3,4 & $-6,339,412$ & $-6,363,803$ & -7989 & $-32,772$ & -2286 & $-27,069$ \\
\hline 3,4 & 1 & $-7,923,820$ & $-7,948,078$ & -9574 & $-34,356$ & -2444 & $-27,227$ \\
\hline 3,4 & 2 & $-7,923,687$ & $-7,948,078$ & -9574 & $-34,356$ & -2444 & $-27,227$ \\
\hline
\end{tabular}

Table 5

Nonlinear Richardson iterations for the solution of the benchmark problem as a function of the Reynolds number and for different sets of interface equations. For the case of the continuity of mean total normal stress, the results obtained by both considering or neglecting the kinetic contribution in the partial derivatives of the Jacobian matrix are both presented.

\begin{tabular}{lllll}
\hline Reynolds number & 1 & 500 & 1000 & 10,000 \\
\hline Mean normal stress & 1 & 1 & 1 & 1 \\
Mean total normal stress & 2 & 3 & 3 & 3 \\
Mean total normal stress (approximated) & 3 & 4 & 4 & 5 \\
\hline
\end{tabular}


As expected, for low Reynolds numbers both solutions are very close, since the kinetic term is nearly negligible with respect to the mean normal stress. On the contrary, for high Reynolds numbers the kinetic term dominates, such that the continuity of the mean normal stress cannot be considered a good approximation of the continuity of the mean total normal stress any longer.

The number of iterations required to obtain the solution of the steady state problem for the different conditions is summarized in Table 5. In the case of continuity of mean normal stress, the Newton method converges in one iteration, since the interface equations and the fluid flow problem defined inside each pipe is linear. In contrast, when imposing the continuity of the mean total normal stress, the number of iterations increases due to the nonlinearity in the interface problem introduced by the kinetic term. However, this increase is bounded to one or two iterations; moreover, it does not show a significant dependence from the Reynolds number for the configuration considered here. We can also observe that if the approximated Jacobian (from the system of equations involving the mean normal stress) is used when imposing the continuity of the mean total normal stress, a slower converge occurs for Reynolds numbers above 1000, when the kinetic term dominates over the hydrostatic pressure term at coupling interfaces.

Finally, the relative CPU time is presented in Table 6. From the first row, which is associated to the continuity of the mean normal stress, we can observe that the CPU time reduces increasing the Reynolds number. This is due to the cost of the evaluation of the residual, which in turn is associated to the cost of solving the linear system for which our preconditioner behaves better in case of low viscosity values. The same behavior is visible in the last two rows, which are associated to the imposition of the continuity of the mean total normal stress. There, however, the cost of the additional iterations required to solve the interface problem inverts the trend for high Reynolds numbers.

\section{Table 6}

Relative CPU time required for the solution of the benchmark problem as a function of the Reynolds number and for different sets of interface equations. For the case of the continuity of mean total normal stress, the results obtained by either considering or neglecting the kinetic contribution in the partial derivatives of the Jacobian matrix are both presented.

\begin{tabular}{|c|c|c|c|c|c|}
\hline Reynolds number & 1 & 500 & 1000 & 5000 & 10,000 \\
\hline Mean normal stress & 1.00 & 0.67 & 0.61 & 0.58 & 0.55 \\
\hline Mean total normal stress & 1.55 & 1.33 & 1.32 & 1.17 & 1.20 \\
\hline Mean total normal stress (approximated) & 1.95 & 1.68 & 1.63 & 1.74 & 2.10 \\
\hline
\end{tabular}

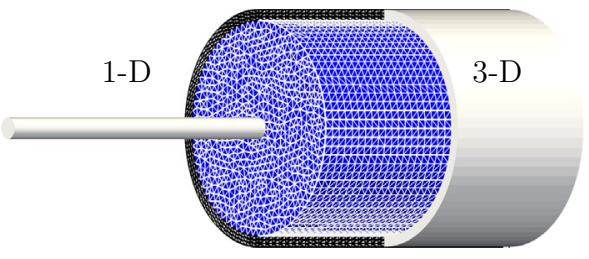

(a)
$1-\mathrm{D}$

(b)

Fig. 8. Schematic representation of the benchmark case. (a) The wave flow propagates from the 1-D FSI model to the 3-D FSI one. The length of each pipe is equal to 3. (b) The reference case: a single 1-D FSI pipe of length 6.

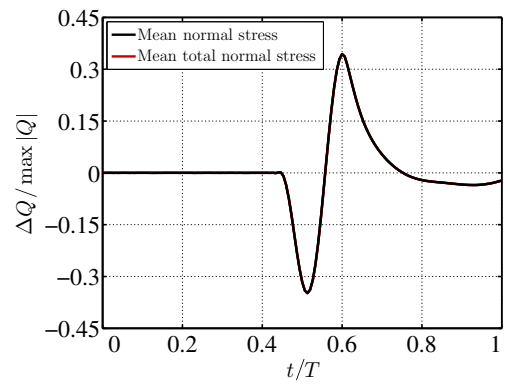

(a)

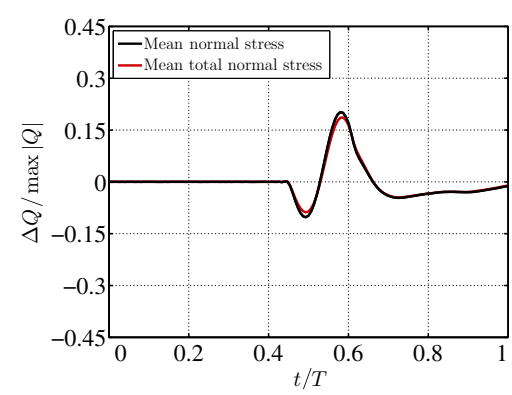

(b)

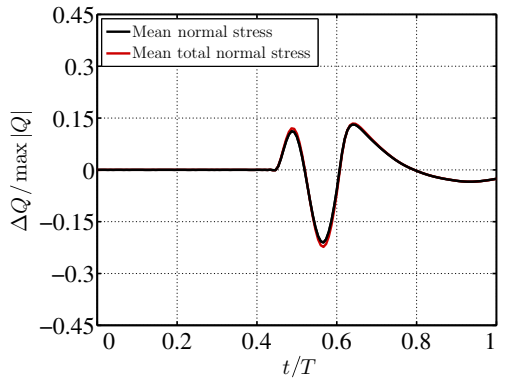

(c)

Fig. 9. Volumetric flow rate wave reflection at the coupling interfaces for different sets of interface equations. On the $x$-axis we represent the normalized time, while on the $y$-axis we show the difference between the volumetric flow rate in the heterogeneous case and the one given by the reference case (see [18, Fig. 5.1]), namely $\Delta Q$, normalized over the maximum amplitude of the inflow wave. This quantity coincides with the normalized reflection wave generated at the coupling interface. (a) Fixed area boundary condition on the 3-D solid ring. (b) Free area boundary condition on the 3-D solid ring. (c) Continuity of the area at the coupling interfaces. 


\subsection{Wave reflection analysis in a series of heterogeneous FSI pipes}

In this section we focus our analysis on the spurious backward wave reflections that might be generated at the coupling interfaces between two dimensionally-heterogeneous pipes. Particularly, we consider a wave that propagates from a 1-D FSI segment to a 3-D FSI pipe (see Fig. 8) and we extend the analysis presented in [18, Section 5] to the case in which the continuity of the mean total normal stress is imposed at the interface between these two pipes. On the leftmost inflow we impose a single flow rate wave defined as

$$
Q(t)= \begin{cases}-\sin \left(\frac{2 \pi t}{T_{\mathrm{w}}}\right)^{2} & t<\frac{T_{\mathrm{w}}}{2} \\ 0 & \text { otherwise }\end{cases}
$$

where $T_{\mathrm{w}}=0.005$ is the chosen wave period. On the rightmost outflow, a classical absorbing boundary condition is applied.
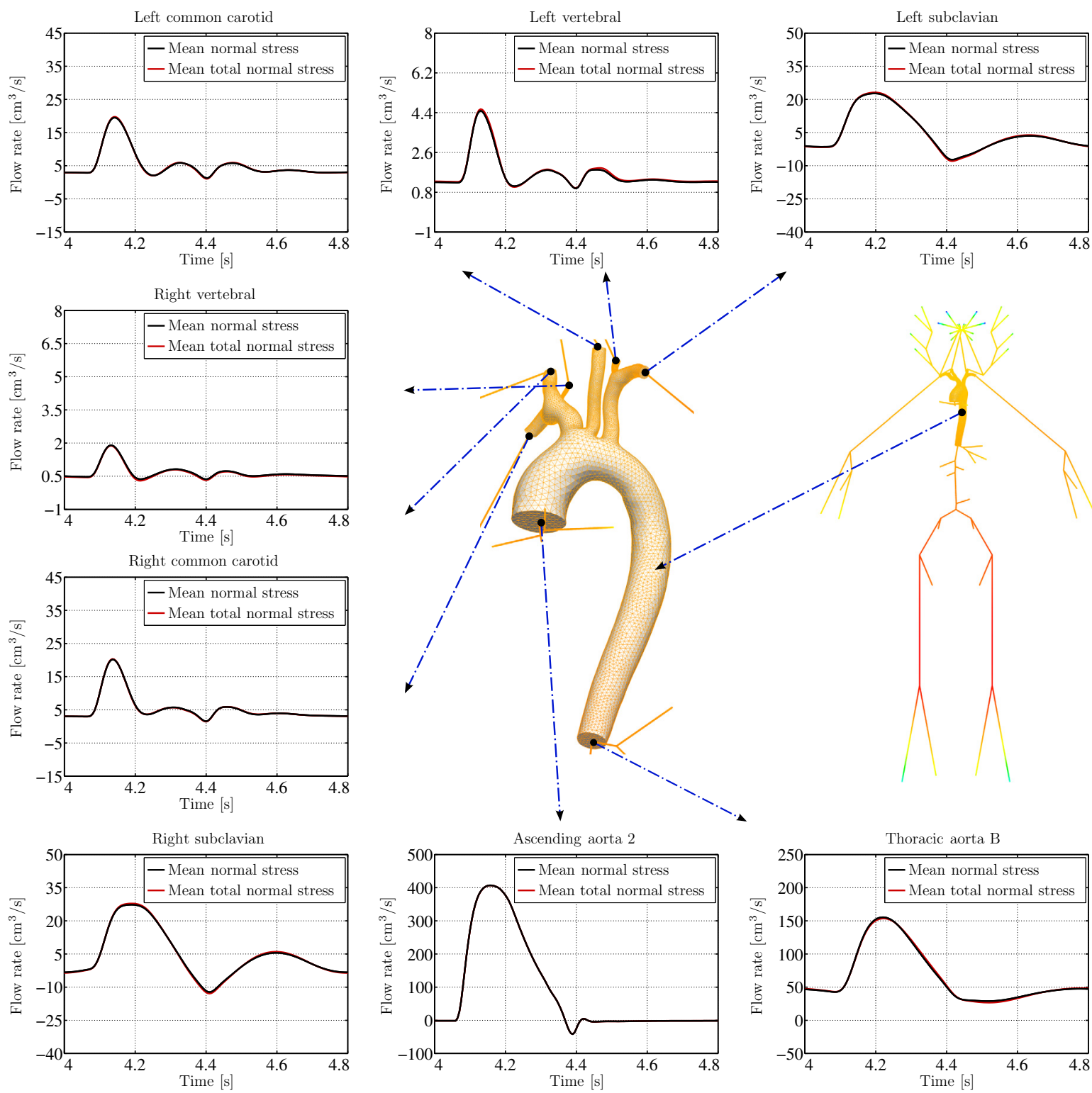

Fig. 10. Flow rate comparison, at the sixth heart beat, between the results obtained by imposing the continuity of the mean normal stress at all the coupling nodes, with respect to those given by enforcing the continuity of the mean total normal stress. The graphs refer to the eight interfaces of the 3-D aorta. The color of the 3-D images represents the pressure field at the end-systole of the sixth heart beat $(t=4.4 \mathrm{~s})$, where the color bar ranges from blue (80,000 dyn/ $\mathrm{cm}^{2}$ ) to red $\left(165,000 \mathrm{dyn} / \mathrm{cm}^{2}\right)$. Positioning of 1-D network elements is purely visual. 
The list of parameters and tolerances, as well as the 3-D fluid and solid mesh sizes used for the numerical simulations are reported in [18, Section 5] and are not repeated here. We remark that, to be coherent with the results presented in [18], at the solid ring interface of the 3-D pipe we impose three different interface conditions corresponding to the case of fixed ring, free ring, and scaled ring (coupled area); see [18, Section 4] for more details about these three interface conditions.
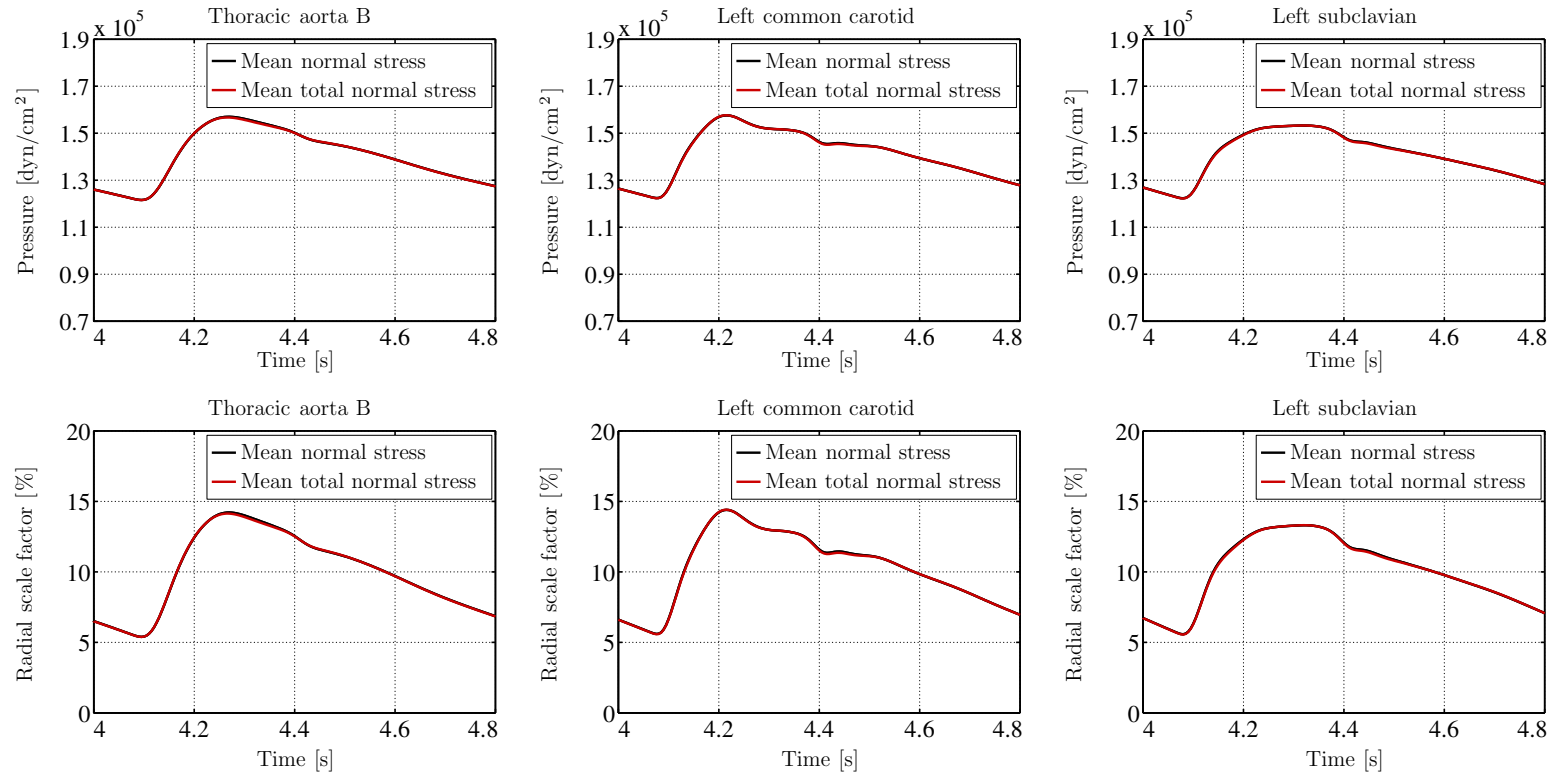

Fig. 11. Pressure and radial scale factor comparisons, at the sixth heart beat, between the results obtained by imposing the continuity of the mean normal stress at all the coupling nodes, with respect to those given by enforcing the continuity of the mean total normal stress. The graphs refer to the most significant coupling interfaces of the 3-D aorta (see Fig. 10).

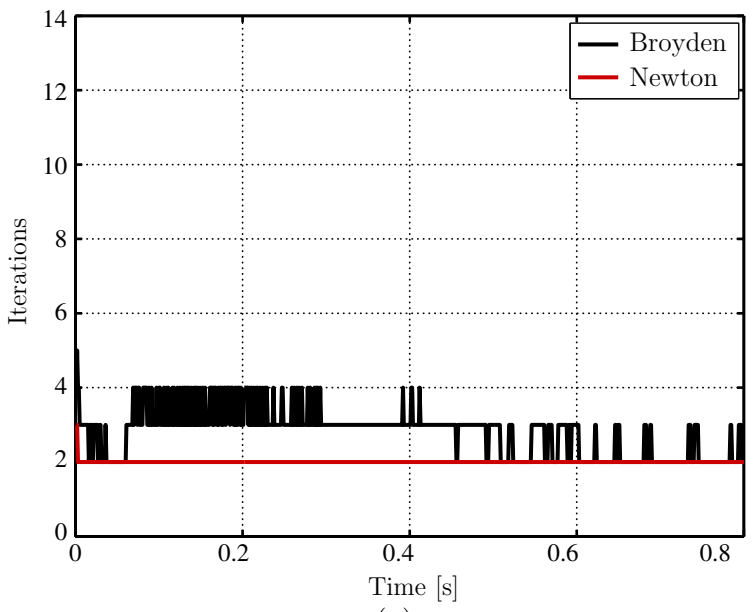

(a)

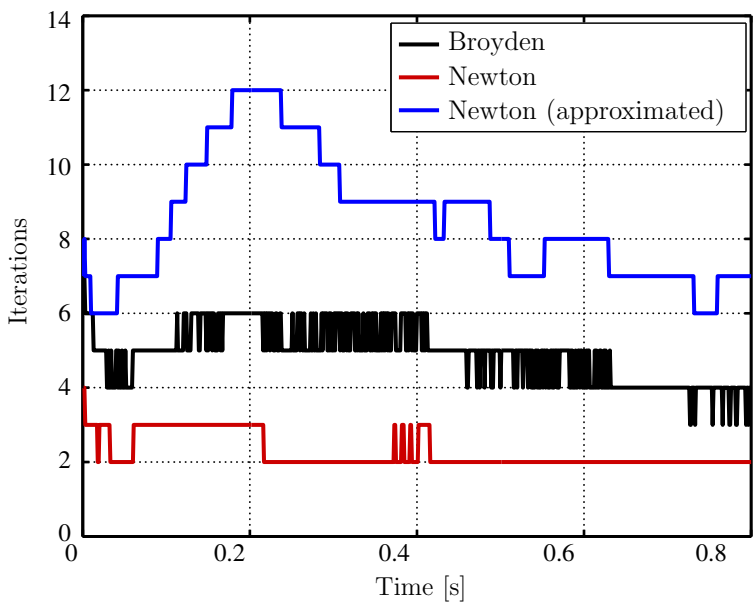

(b)

Fig. 12. Comparison, in terms of number of iterations, of different algorithms for the coupling of the geometrical multiscale model at the first heart beat. (a) Continuity of the mean normal stress imposed at all the interfaces. (b) Continuity of the mean total normal stress imposed at all the interfaces. Recall that continuity of the mean normal stress is imposed at interfaces between 1-D and windkessel models.

Table 7

Relative CPU time required for the solution of the first heart beat of the full geometrical multiscale model.

\begin{tabular}{|c|c|c|c|c|c|}
\hline & \multicolumn{2}{|c|}{ Mean normal stress } & \multicolumn{3}{|c|}{ Mean total normal stress } \\
\hline & Broyden & Newton & Broyden & Newton & Newton (approximated) \\
\hline Relative CPU time per heart beat & 1.00 & 5.41 & 1.51 & 5.77 & 20.44 \\
\hline
\end{tabular}


The comparison between the imposition of the continuity of the mean normal stress (see problem (7)), with that of the mean total normal stress (see problem (10)) for a wave that propagates from a 1-D FSI segment to a 3-D FSI pipe (see [18, Fig. 5.1]) is presented in Fig. 9. The same result (which for brevity is not reported here) holds in the inverted configuration (wave propagation from a 3-D FSI pipe to a 1-D FSI segment, see [18, Fig. 5.5]).

The difference between the results computed with the two sets of interface equations is very small because, for the chosen set of values of the parameters, the kinetic contribution to the composition of the mean total normal stress is almost negligible. This is also characteristic in the flow regimes observed when studying blood circulation, as shown in the next section.

Therefore, we can conclude that the imposition of the mean total normal stress does not affect the numerical reflections that emerge at coupling interfaces of the problem. Regarding the convergence properties, we postpone our analysis to the next section, where we study a problem characterized by several interfaces.

\subsection{Geometrical multiscale modeling: 3-D FSI aorta coupled with 1-D FSI network}

In this section we use one of the geometrical multiscale models presented in [19] to compare, in a realistic cardiovascular setting, the results of the set of interface equations imposing the continuity of the mean normal stress, with those of the interface equations prescribing the continuity of the mean total normal stress. Specifically, as benchmark we select the geometrical multiscale model composed by assembling a large network of 1-D arteries (see [19, Section 3.1]) with a 3-D geometry representing the aorta (see [19, Fig. 2(a)]). The full detailed description of the benchmark, the list of parameters and tolerances, as well as the 3-D fluid and solid mesh sizes used for the numerical simulations are reported in the reference work and are not repeated here. We just remark that, among the several sets of values for the elastic external tissues parameters presented in [19, Section 3.3.1], we select the one named $E_{4}^{A}$, while the viscoelastic ones are determined through the empiric relation $c_{\mathrm{S}}=k_{\mathrm{S}} / 10$ [19].

In all the presented cases we impose the continuity of the vessel area at the interfaces between the 3-D geometries and the 1-D arteries through either (15) or (16). Moreover, the continuity of the mean normal stress is always imposed at the coupling nodes between the distal 1-D segments and the corresponding windkessel terminal models.

The results of the flow rate waveform comparison at all the coupling interfaces between the 1-D network and the 3-D aorta are summarized in Fig. 10. As expected, the results of the two sets of interface equations almost coincide. This behavior can be justified by the fact that the kinetic contribution to the mean total normal stress is negligible compared to the one given by the pressure; in particular, in a physiological regime, the ratio between the pressure and the kinetic term is around one hundred. Regarding the behavior of the pressure and the radial scale factor, which are summarized in Fig. 11, similar comments hold.

Finally, in Fig. 12 we compare the number of iterations required by different algorithms for the solution of the global geometrical multiscale problem, for both sets of interface equations. When imposing the continuity of the mean normal stress at all the nodes of the problem we need an average of 2 iterations per time step to achieve convergence using the Newton method, while this number increases to 2.8 when employing the Broyden method. In contrast, when imposing the continuity of the mean total normal stress, the average number of iterations per time step increases to 2.26 and to 4.89 for the Newton and Broyden methods, respectively. Therefore, we conclude that despite the physiological results obtained for the two sets of interfaces equations are very similar, the imposition of the continuity of the mean total normal stress leads to an increase of the computational cost of the problem, especially when using the Broyden method. Moreover, in the case where the Jacobian matrix is approximated by neglecting the kinetic contribution, the performance of the Newton method strongly deteriorates, requiring an average of 8.48 iterations per time step.

Notice that from the point of view of the computational cost, the Broyden method is still the cheapest one (see Table 7). Indeed, it does not require the solution of the tangent problem associated to the 3-D FSI model (see [[18], Section 6] for another quantitative example). In particular, we remark that to assemble the Jacobian matrix at each Newton iteration the algorithm requires the solution of a 3-D tangent problem for each interface of a 3-D model: since the 3-D aorta considered here has eight interfaces, the approximate number of tangent problems solved during each time step is 18, which entails a significant computational cost.

The comparisons between the solutions obtained for the two different sets of interface equations inside the aorta, in terms of 3-D velocity and pressure fields, have been omitted because the results practically coincide; this is a direct consequence of, and can be concluded from, the indistinctness of results reported in Fig. 11.

\section{Conclusions}

In the present work a methodology to couple dimensionally-heterogeneous models in an iterative fashion by imposing the continuity of the mean total normal stress at the interfaces has been proposed and tested. This has been carried out by extending a geometrical multiscale framework in order to account for this new interface equation, while maintaining robustness and flexibility in the existing algorithms. The salient feature of the proposed strategy is that it makes possible to preserve the total energy at model interfaces while imposing Neumann boundary conditions in the classical Navier-Stokes formulation of the 3-D FSI model. 
The new set of energy preserving interface equations has been tested over several examples and its results have been compared with those provided by the original approach, which preserves the continuity of the mean normal stress. For both strategies convergence properties of Newton and Broyden methods have been assessed for different flow regimes. It is concluded that both, Newton and Broyden methods are robust alternatives to solve the associated system of interface equations, while the former performs better in terms of iterations number and the latter performs better in terms of overall computational cost.

Moreover, it is worthwhile to highlight that the results obtained at flow regimes observed in the cardiovascular system by using the continuity of the mean total normal stress are practically the same to those obtained when using the continuity of the mean normal stress. Indeed, no unstable conditions caused by the lack of an energy boundedness theoretical result were observed in the latter case, neither in the simulations reported here, nor in those conducted in our previous works and that were not repeated here. This is important in practical applications because prescribing the continuity of the mean normal stress is much simpler at the software level and implies less computational cost, as also demonstrated in the examples conducted in this work.

In conclusion, we believe that continuity of mean normal stress can be safely used for most of the cardiovascular applications. Nonetheless, the presented coupling algorithm allows the user to decide whether to consider the continuity of the mean total normal stress or the continuity of the mean normal stress, without having to be concerned with modifications of features which are specific of the computational models.

\section{Acknowledgements}

P.J. Blanco acknowledges the support of the Brazilian agencies CNPq and FAPERJ. A.C.I. Malossi, and S. Deparis acknowledge the European Research Council Advanced Grant "Mathcard, Mathematical Modelling and Simulation of the Cardiovascular System", Project ERC-2008-AdG 227058, and the Swiss Platform for High-Performance and High-Productivity Computing (HP2C). We also acknowledge the support of the Brazilian-Swiss Joint Research Programme (BSJRP), Project BJRP 011010 (N 590002/2010-4). All the numerical results presented in this paper have been computed using the [ofortt]Li feV[cfortt] library (www.lifev.org).

\section{References}

[1] P.J. Blanco, M.R. Pivello, S.A. Urquiza, R.A. Feijóo, On the potentialities of 3D-1D coupled models in hemodynamics simulations, J. Biomech. 42 (2009) 919-930.

[2] P.J. Blanco, S.A. Urquiza, R.A. Feijóo, Assessing the influence of heart rate in local hemodynamics through coupled 3D-1D-0D models, Int. J. Numer. Methods Biomed. Eng. 26 (2010) 890-903.

[3] J. Bonnemain, A.C.I. Malossi, M. Lesinigo, S. Deparis, A. Quarteroni, L.K. von Segesser, Numerical simulation of left ventricular assist device implantations: comparing the ascending and the descending aorta cannulations, Med. Eng. Phys. (2013). Available from: http://dx.doi.org/10.1016/ j.medengphy.2013.03.022.

[4] L. Formaggia, F. Nobile, A. Quarteroni, A. Veneziani, Multiscale modelling of the circulatory system: a preliminary analysis, Comput. Visual. Sci. 2 (1999) $75-83$.

[5] L. Grinberg, E. Cheever, T. Anor, J.R. Madsen, G.E. Karniadakis, Modeling blood flow circulation in intracranial arterial networks: a comparative 3D/1D simulation study, Ann. Biomed. Eng. 39 (2011) 297-309.

[6] H.J. Kim, I.E. Vignon-Clementel, C.A. Figueroa, J.F. LaDisa, K.E. Jansen, J.A. Feinstein, C.A. Taylor, On coupling a lumped parameter heart model and a three-dimensional finite element aorta model, Ann. Biomed. Eng. 37 (2009) 2153-2169.

[7] T. Lassila, A.C.I. Malossi, M. Stevanella, E. Votta, A. Redaelli, S. Deparis, Multiscale fluid-structure interaction simulation of anatomically correct left ventricle fluid dynamics with fictitious elastic structure regularization, 2012, submitted for publication.

[8] F. Migliavacca, R. Balossino, G. Pennati, G. Dubini, T.Y. Hsia, M.R. de Leval, E.L. Bove, Multiscale modelling in biofluidynamics: application to reconstructive paediatric cardiac surgery, J. Biomech. 39 (2006) 1010-1020.

[9] L. Formaggia, J.-F. Gerbeau, F. Nobile, A. Quarteroni, On the coupling of 3D and 1D Navier-Stokes equations for flow problems in compliant vessels, Comput. Meth. Appl. Mech. Eng. 191 (2001) 561-582.

[10] P.J. Blanco, R.A. Feijóo, S.A. Urquiza, A unified variational approach for coupling 3D-1D models and its blood flow applications, Comput. Meth. Appl. Mech. Eng. 196 (2007) 4391-4410.

[11] L. Formaggia, A. Quarteroni, C. Vergara, On the physical consistency between three-dimensional and one-dimensional models in haemodynamics, J. Comput. Phys. 244 (2013) 97-112.

[12] H.J. Kim, C.A. Figueroa, T.J.R. Hughes, K.E. Jansen, C.A. Taylor, Augmented Lagrangian method for constraining the shape of velocity profiles at outlet boundaries for three-dimensional finite element simulations of blood flow, Comput. Meth. Appl. Mech. Eng. 198 (2009) $3551-3566$.

[13] M.E. Moghadam, Y. Bazilevs, T.-Y. Hsia, I.E. Vignon-Clementel, A.L. Marsden, A comparison of outlet boundary treatments for prevention of backflow divergence with relevance to blood flow simulations, Comput. Mech. 48 (2011) 277-291.

[14] L. Formaggia, A. Moura, F. Nobile, On the stability of the coupling of 3D and 1D fluid-structure interaction models for blood flow simulations, ESAIM: Math. Model. Numer. Anal. 41 (2007) 743-769.

[15] F. Nobile, Coupling strategies for the numerical simulation of blood flow in deformable arteries by 3D and 1D models, Math. Comput. Model. 49 (2009) $2152-2160$

[16] V. Gravemeier, A. Comerford, L. Yoshihara, M. Ismail, W.A. Wall, A novel formulation for Neumann inflow boundary conditions in biomechanics, Int. J. Numer. Methods Biomed. Eng. 28 (2012) 560-573.

[17] A.C.I. Malossi, P.J. Blanco, S. Deparis, A two-level time step technique for the partitioned solution of one-dimensional arterial networks, Comput. Meth. Appl. Mech. Eng. 237-240 (2012) 212-226.

[18] A.C.I. Malossi, P.J. Blanco, P. Crosetto, S. Deparis, A. Quarteroni, Implicit coupling of one-dimensional and three-dimensional blood flow models with compliant vessels, SIAM J. Multiscale Model. Simul. 11 (2013) 474-506.

[19] A.C.I. Malossi, J. Bonnemain, Numerical comparison and calibration of geometrical multiscale models for the simulation of arterial flows, Cardiovasc. Eng. Technol. 4 (2013). Available from: http://dx.doi.org/10.1007/s13239-013-0151-9.

[20] P. Crosetto, Fluid-structure interaction problems in hemodynamics: parallel solvers, preconditioners, and applications, Ph.D. thesis, École Polytechnique Fédérale de Lausanne, 2011. 
[21] P. Crosetto, S. Deparis, G. Fourestey, A. Quarteroni, Parallel algorithms for fluid-structure interaction problems in haemodynamics, SIAM J. Sci. Comput. 33 (2011) 1598-1622.

[22] E. Burman, M.A. Fernández, P. Hansbo, Continuous interior penalty finite element method for Oseen’s equations, SIAM J. Numer. Anal. 44 (2006) 1248 1274.

[23] A. Quarteroni, L. Formaggia, Mathematical modelling and numerical simulation of the cardiovascular system, in: N. Ayache (Ed.), Computational Models for the Human Body, Handbook of Numerical Analysis, vol. 12, Elsevier, 2004, pp. 3-127.

[24] A.C.I. Malossi, P.J. Blanco, S. Deparis, A. Quarteroni, Algorithms for the partitioned solution of weakly coupled fluid models for cardiovascular flows, Int. J. Numer. Methods Biomed. Eng. 27 (2011) 2035-2057.

[25] J.S. Leiva, P.J. Blanco, G.C. Buscaglia, Partitioned analysis for dimensionally-heterogeneous hydraulic networks, SIAM J. Multiscale Model. Simul. 9 (2011) 872-903.

[26] P.J. Blanco, J.S. Leiva, G.C. Buscaglia, A black-box decomposition approach for coupling heterogeneous components in hemodynamics simulations, Int. J. Numer. Methods Biomed. Eng. 29 (2013) 408-427.

[27] A.C.I. Malossi, Partitioned solution of geometrical multiscale problems for the cardiovascular system: models, algorithms, and applications, Ph.D. thesis, École Polytechnique Fédérale de Lausanne, 2012. 